\title{
Characteristics of wall pressure fluctuations for a flat plate turbulent boundary layer with pressure gradients
}

\author{
Nan $\mathrm{Hu}^{*}$ and Michaela Herr ${ }^{\dagger}$ \\ DLR, Technical Acoustics Branch, D-38108 Braunschweig, Germany
}

\begin{abstract}
The wall pressure fluctuations beneath a turbulent boundary layer with zero and non-zero pressure gradients were measured at a flat plate configuration in the Acoustic Windtunnel Braunschweig. The fluctuating pressure was measured by an array of subminiature pressure transducers. In addition, the mean flow velocity profiles within the turbulent boundary layer were obtained using hot wires. Adverse and favorable pressure gradients were realized by placing a rotatable NACA 0012 airfoil with a chord length of $40 \mathrm{~cm}$ above the flat plate. The one-point spectra and the two-point correlation properties are analysed. An empirical spectral model for the wall pressure fluctuations beneath an adverse pressure gradient boundary layer is developed based on the measured data. The effects of the pressure gradients on the characteristics of the wall pressure fluctuations are discussed.
\end{abstract}

\section{Introduction}

Wall pressure fluctuations beneath a turbulent boundary layer have been extensively studied in theoretical, experimental and numerical studies in the last decades. The major concern is the flow-induced vibration and the resulting sound generation. In general, not only the excitation power but also the spatial and temporal properties of the wall pressure fluctuations are relevant for the resulting vibration. The correlation decay in longitudinal and lateral directions and the convective velocity of the surface fluctuating pressure pattern are the most relevant features for representing the spatial and temporal properties. A comprehensive overview on the subject of wall pressure fluctuations, the structural response and the induced sound radiation was given in the monograph of Blake. ${ }^{1}$ Several fundamental experiments have been carried out to measure the characteristics of the wall pressure fluctuations, e.g. refer to the work of Willmarth \& Wooldridge, Bull, Blake and Farabee \& Casarella. ${ }^{2-5}$ Many empirical spectral models were proposed. One of the most frequently cited models is the one from Goody, ${ }^{6}$ which was derived from experimental results for zero pressure gradient (ZPG) turbulent boundary layers. To represent the spatial and temporal properties of the fluctuating pressure field, $\operatorname{Cor} \cos ^{7}$ proposed exponential functions based on empirical coherence decay parameters of both longitudinal and lateral directions and the phase velocities.

However, most studies are restricted to ZPG boundary layers. Schloemer ${ }^{8}$ was the first to measure the wall pressure fluctuations under both adverse and favorable pressure gradients. Several important knowledge was gained from that experiment. For example, compared to the ZPG boundary layers, the ratio between the convective velocity and the freestream velocity $U_{c} / U_{0}$ is smaller for the adverse pressure gradient (APG) boundary layers and larger for the favorable pressure gradient (FPG) boundary layers. The coherence decay in longitudinal direction is faster for the APG and slower for the FPG boundary layers. In recent work, Rozenberg et al. ${ }^{9}$ (RRM) proposed an empirical spectral model for APG boundary layers based on existing experimental and numerical results. Catlett et al. ${ }^{10,11}$ (CFAS) measured the wall pressure fluctuations on airfoil trailing-edge configurations with varying opening angles and proposed an empirical spectral model based on the measured data. They both modified the Goody model using different boundary-layer characteristics as input quantities. Catlett et al. showed an unsatisfactory prediction for their measured surface pressure data using the RRM model. Suryadi and Herr ${ }^{12}$ applied both RRM and CFAS models to predict their measured data on a DU96-W-180 airfoil. However, the results of both models showed large discrepancies compared to the measured data.

${ }^{*}$ Research Engineer, AIAA member, nan.hu@dlr.de

${ }^{\dagger}$ Research Engineer, AIAA senior member, michaela.herr@dlr.de 
Among others, Clauser's equilibrium parameter ${ }^{13}$ defined as $\beta_{\delta^{*}}=\left(\delta^{*} / \tau_{w}\right)(d p / d x),{ }^{14}$ is used as one important input quantity in both RRM and CFAS models, i. e. the local pressure gradient, normalized with the wall shear stress and the boundary-layer displacement thickness. Equilibrium flows hold a constant $\beta_{\delta^{*}}$, e.g. $\beta_{\delta^{*}}=0$ for the specific case of a ZPG boundary layer. ${ }^{13,14}$ For equilibrium boundary layers Clauser demonstrated a clear dependence of the velocity profile shapes on this single parameter. Building upon Clauser's work, Mellor and Gibson ${ }^{14}$ accomplished to predict the measured equilibrium defect profiles from Clauser. Herring and Norbury ${ }^{15}$ performed supplementing experiments on FPG equilibrium boundary layers, where $\beta_{\delta^{*}}$ possess negative values. A good representation of their measured FPG velocity profiles was achieved using the theory of Mellor and Gibson. Accordingly, for equilibrium flows selection of $\beta_{\delta^{*}}$ is considered a well-suited parameter to reproduce the effect of a non-zero pressure gradient on the velocity profile and corresponding surface pressure spectra.

In the present work it is hypothesized that the shape parameter $H=\delta^{*} / \theta$ might represent a better suited nondimensional quantity to cope with the prediction of surface pressures under arbitrary nonequilibrium flow conditions, where also the history of the boundary-layer development is considered important. In the following, the results from measurements of the wall pressure fluctuations on a flat plate for ZPG, APG and FPG boundary layers are presented. The effects of pressure gradients on the one-point spectra, the two-point correlations and the convective properties are discussed. In addition, a new empirical spectral model is developed for the prediction of the wall pressure fluctuations beneath APG boundary layers. The results from the new model are compared to the present measured data and to other results from literature.

\section{Experimental setup}

The experiment was conducted in the open-jet anechoic test section of the Acoustic Windtunnel Braunschweig (AWB). The wind tunnel has a rectangular nozzle with a height of $1200 \mathrm{~mm}$ and a width of $800 \mathrm{~mm}$. The maximum operating velocity is $U_{0}=65 \mathrm{~m} / \mathrm{s}$. Details of the experimental setup are documented in Figs. 1 and 2. A flat wooden plate was placed $10 \mathrm{~mm}$ downstream of the nozzle exit in the mid-height nozzle position. The plate surface was aligned with the flow direction. The plate span is $1300 \mathrm{~mm}$ which is $250 \mathrm{~mm}$ wider than the nozzle exit on each side to prevent side-edge interaction with the AWB open-jet shear-layers, see Fig. 1(c). The length and thickness of the plate are $1350 \mathrm{~mm}$ and $42 \mathrm{~mm}$, respectively. A $125 \mathrm{~mm}$ long superellipse $(n=3)$ shaped leading-edge part was selected to avoid flow separation ${ }^{16}$ and manufactured by 3D printing. Both sides of the plate were tripped at $120 \mathrm{~mm}$ behind the leading edge tip with $0.3 \mathrm{~mm}$ zigzag trip strips. A $12^{\circ}$ beveled trailing edge on the underside of the plate was used to realize a ZPG turbulent boundary-layer on the topside in the rear area. ${ }^{17}$ The 5 -mm thick trailing-edge tip was extended by foam serrations to avoid vortex shedding and to reduce trailing-edge noise.

A $370 \mathrm{~mm}$ long, $270 \mathrm{~mm}$ wide and $5 \mathrm{~mm}$ thick aluminium panel equipped with 25 static pressure ports and twelve Kulite pressure transducers was placed at mid-span in the rear portion of the plate. The rear edge of the panel was located at $90 \mathrm{~mm}$ upstream of the trailing edge of the plate. The static pressure ports covered $290 \mathrm{~mm}$ in streamwise direction and $180 \mathrm{~mm}$ in spanwise direction. The wall pressure fluctuations were measured by twelve pinhole-mounted Kulite pressure transducers without the protection screen, model LQ-062-0.35bar. The diameter of the pinhole was $0.5 \mathrm{~mm}$ and the depth was $0.5 \mathrm{~mm}$. The Kulite sensor with a diameter of $1.6 \mathrm{~mm}$ was glued with silicone in a $1.8 \mathrm{~mm}$ diameter hole behind the pinhole. The Kulite sensors were located in streamwise direction between $1128 \mathrm{~mm} \leq x \leq 1210 \mathrm{~mm}$ $(x=0$ for the leading edge tip of the plate) and in spanwise direction between $0 \mathrm{~mm} \leq y \leq 27 \mathrm{~mm}$ $(y=0$ for the mid-span). The layout for the Kulites and the static pressure ports on the panel are shown in Fig. 3. During the measurement the sampling rate was set at $100 \mathrm{kHz}$ and the data were recorded for $20 \mathrm{~s}$. A preamplifier with a gain factor of 250 and a high pass filter with cut-off frequency at $200 \mathrm{~Hz}$ was applied. The measured power spectra shown in this paper are corrected using the filter frequency response curve.

Pressure gradients were realized by placing a rotatable NACA 0012 airfoil with $400 \mathrm{~mm}$ chord length and $1800 \mathrm{~mm}$ span width above the plate. The airfoil was installed with the chord position $120 \mathrm{~mm}$ above the plate at the geometric angle of attack of $0^{\circ}$. The rotation axis was at $41 \%$ of the chord length. The geometric angle of attack of the airfoil was varied between $-14^{\circ}$ and $14^{\circ}$. The leading edge of the airfoil was located at $x=850 \mathrm{~mm}$. Both sides of the airfoil were tripped at $20 \%$ chord length with $0.4 \mathrm{~mm}$ zigzag trip strips. Static pressure distributions of the NACA 0012 airfoil were measured with 46 static pressure ports.

Velocity profiles within the turbulent boundary layer were measured by single hot-wire anemometry. The hot-wire data were recorded for $10.3 \mathrm{~s}$ with a sampling rate of $50 \mathrm{kHz}$ and a low pass filter of $20 \mathrm{kHz}$. 


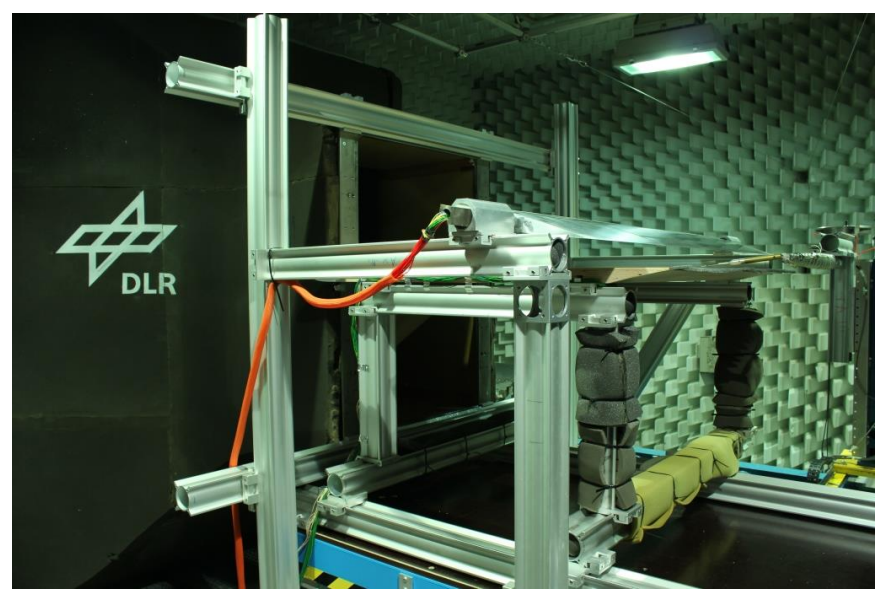

(a)

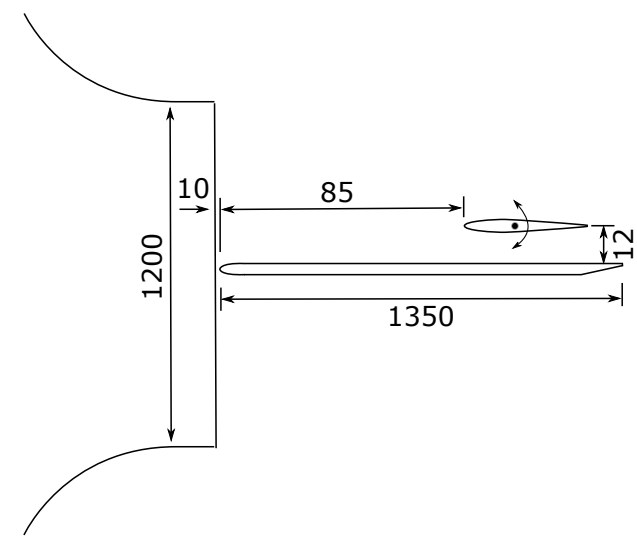

(b)

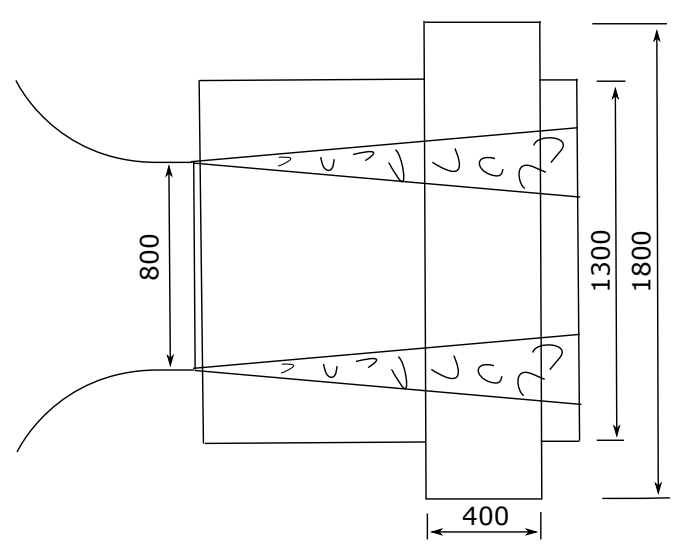

(c)

Figure 1: (a) Experimental setup in AWB; (b) schematic side view; (c) schematic top view.

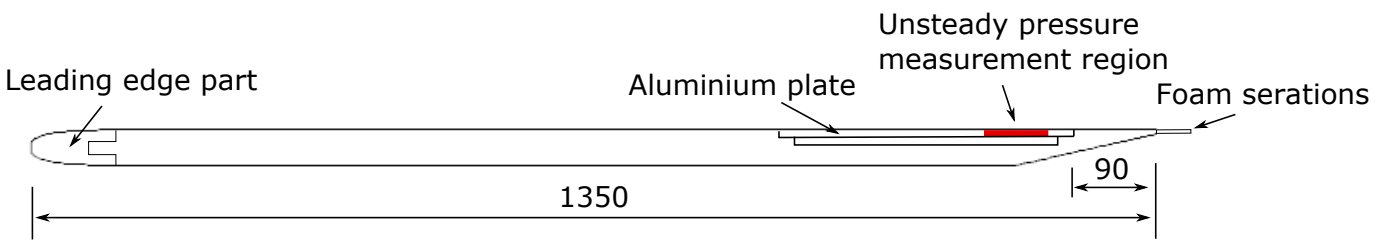

Figure 2: Schematic view of the plate configuration.

\section{Results}

\section{III.A. Mean flow characteristics}

The mean velocity profiles for the ZPG cases were measured at $x=1210 \mathrm{~mm}$ where the most downstream Kulite sensors were located. Five test velocities between $20 \mathrm{~m} / \mathrm{s}<U_{0}<59 \mathrm{~m} / \mathrm{s}$ were selected for ZPG flow measurements. Spanwise measurements confirmed an approximate $500-\mathrm{mm}$ extent of uniform $2 \mathrm{D}$ flow conditions at $x=1210 \mathrm{~mm}$. ZPG conditions in the current study effectively correspond to weak APG conditions, i. e. to $d C_{p} / d x \leq 0.1 \mathrm{~m}^{-1}$ between $930 \mathrm{~mm} \leq x \leq 1220 \mathrm{~mm}$ (refer also to Fig. 5).

Fig. 4 shows the measured ZPG mean velocity profiles for all test velocities. The mean velocities for locations $y<1.5 \mathrm{~mm}$ are estimated using Spalding's equation, ${ }^{18}$

$$
y^{+}=u^{+}+e^{-\kappa B}\left[e^{\kappa u^{+}}-1-\kappa u^{+}-\frac{\left(\kappa u^{+}\right)^{2}}{2}-\frac{\left(\kappa u^{+}\right)^{3}}{6}\right],
$$

where $u^{+}=u / u_{\tau}$ and $y^{+}=y u_{\tau} / \nu$. The friction velocity $u_{\tau}$ is obtained by fitting the measurement 


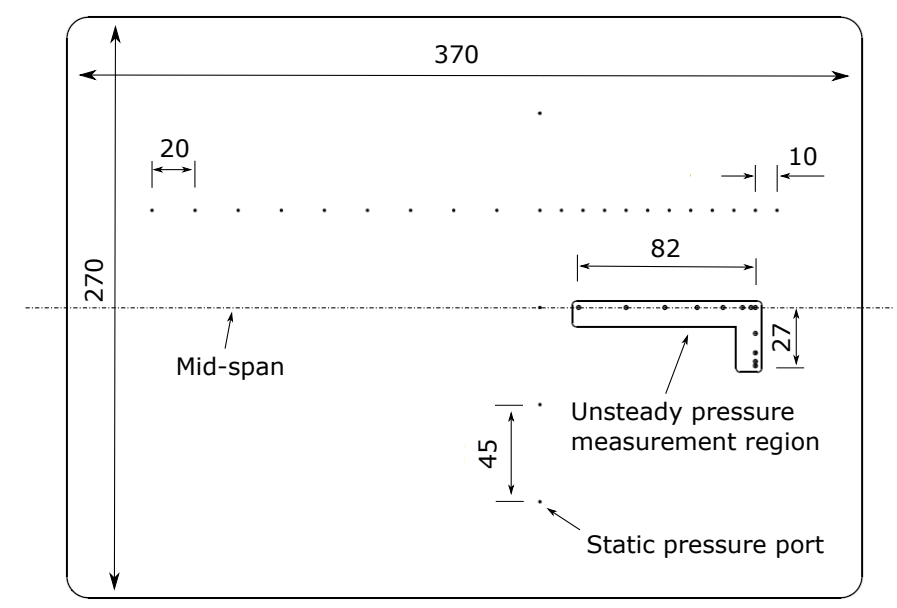

Figure 3: Layout for the Kulites and the static pressure ports on the aluminium panel.

data to the log-law region. The present estimate applies constant $\kappa=0.41$ and $B=5.0 .{ }^{19}$ Spalding's formula comprises all boundary layer regions in one single expression and provides an excellent fit from the sublayer to the log-law region. ${ }^{20}$

When normalized with the outer flow parameters $U_{0}$ and the boundary layer thickness $\delta$, Fig. 4(a), the profiles for $U_{0} \geq 39.2 \mathrm{~m} / \mathrm{s}$ exhibit identical shapes, whereas the lower Reynolds number cases $(20.3 \mathrm{~m} / \mathrm{s}$ and $30.2 \mathrm{~m} / \mathrm{s}$ ) slightly deviate. The relevant characteristic parameters for the ZPG boundary layers are listed in Table 1. Accordingly, the observed scatter of the normalized velocity profiles for varying test speeds is well represented by the shape factor $H$, where $H=1.42$ for $20.3 \mathrm{~m} / \mathrm{s}$ and $H=1.41$ for $30.2 \mathrm{~m} / \mathrm{s}$ are a little larger than $H=1.37-1.38$ for the higher velocities. Following Clauser, ${ }^{13} H$ is a function of $R e_{x}$ for equilibrium boundary layers, e.g. for $10^{6}<R e_{x}<10^{7}$ ZPG values of $H$ are expected to vary between $1.26-1.35$. Nikuradse ${ }^{21}$ measured the boundary layer for pipe flow in a range of $1.7 \cdot 10^{6} \leq R e_{x} \leq 1.8 \cdot 10^{7}$. He found a universal mean velocity distribution which is independent of $R e_{x}$ within the measurement range and derived constant $H=1.3$. The current study indicates a weak $R e_{x}$ dependence. Overall, due to the mild APG in the plate rear region, the obtained values of $H$ are slightly larger than the results from literature.

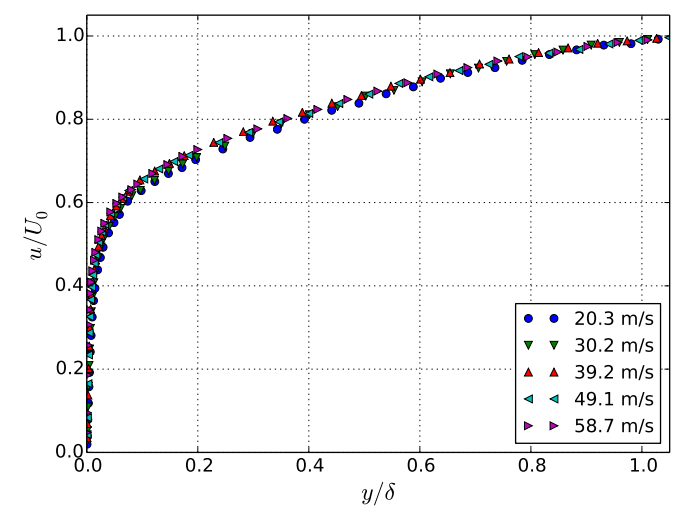

(a)

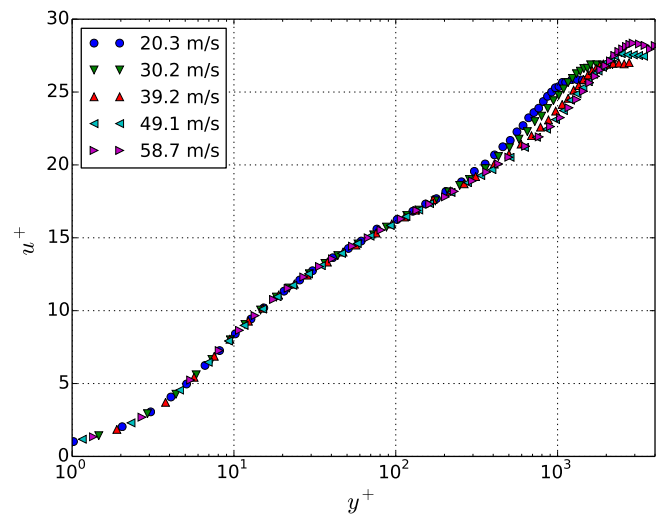

(b)

Figure 4: Boundary layer mean velocity profiles for ZPG at velocities between $20 \mathrm{~m} / \mathrm{s}<U_{0}<59 \mathrm{~m} / \mathrm{s}$, $x=1210 \mathrm{~mm}$.

APG boundary layers were realized by means of the NACA 0012 airfoil at geometric angles of attack of $-6^{\circ},-10^{\circ}$ and $-14^{\circ}$, and FPG boundary layers at $12^{\circ}$ and $14^{\circ}$. The following analysis of the data under pressure gradient is limited to $U_{0}=30.2 \mathrm{~m} / \mathrm{s}$. Fig. 5 shows the measured distributions of the pressure coefficient $C_{p}$ between $930 \mathrm{~mm} \leq x \leq 1220 \mathrm{~mm}$. Velocity profiles were measured at two positions $x=1128 \mathrm{~mm}$ and $x=1210 \mathrm{~mm}$, where the most upstream and downstream Kulite sensors were located. Fig. 6 shows the mean velocity profiles for ZPG, APG and FPG boundary layers at $x=1210 \mathrm{~mm}$. 
Table 1: Boundary layer parameters for ZPG.

\begin{tabular}{ccccccccccc}
\hline \hline $\begin{array}{c}U_{0} \\
(\mathrm{~m} / \mathrm{s})\end{array}$ & $\begin{array}{c}\delta \\
(\mathrm{mm})\end{array}$ & $\begin{array}{c}\delta^{*} \\
(\mathrm{~mm})\end{array}$ & $\begin{array}{c}\theta \\
(\mathrm{mm})\end{array}$ & $H$ & $\begin{array}{c}u_{\tau} \\
(\mathrm{m} / \mathrm{s})\end{array}$ & $\begin{array}{c}R e_{x}= \\
U_{0} x / \nu\end{array}$ & $\begin{array}{c}R e_{\tau}= \\
u_{\tau} \delta / \nu\end{array}$ & $\begin{array}{c}R e_{\theta}= \\
U_{0} \theta / \nu\end{array}$ & $\begin{array}{c}d p / d x \\
(\mathrm{~Pa} / \mathrm{m})\end{array}$ & $\beta_{\delta^{*}}$ \\
\hline 20.3 & 20.4 & 3.8 & 2.67 & 1.42 & 0.785 & $1.6 \cdot 10^{6}$ & 1040 & 3522 & 18 & 0.09 \\
30.2 & 19.7 & 3.51 & 2.49 & 1.41 & 1.125 & $2.4 \cdot 10^{6}$ & 1439 & 4889 & 42 & 0.10 \\
39.2 & 18.8 & 3.15 & 2.28 & 1.38 & 1.455 & $3.1 \cdot 10^{6}$ & 1776 & 5806 & 79 & 0.10 \\
49.1 & 18.5 & 3.15 & 2.29 & 1.38 & 1.78 & $3.9 \cdot 10^{6}$ & 2138 & 7286 & 128 & 0.11 \\
58.7 & 18.5 & 3.13 & 2.28 & 1.37 & 2.08 & $4.6 \cdot 10^{6}$ & 2499 & 8685 & 184 & 0.11 \\
\hline \hline
\end{tabular}

The measured trends show good agreement with the experimental results from literature. ${ }^{20}$ The FPG boundary layer shows a larger velocity increase in the inner layer, when compared to the ZPG case. Contrarily, the APG boundary layer exhibits a steeper velocity increase in the outer layer. For a very strong APG boundary layer, e.g. the APG $-14^{\circ}$ case, an inflection point occurs at the transition region between the inner and outer layer, i. e. at about $0.1-0.2 \delta$. If we plot the profiles in log-law representation, all profiles collapse to a single curve in the inner layer, as shown in Fig. 6(b).

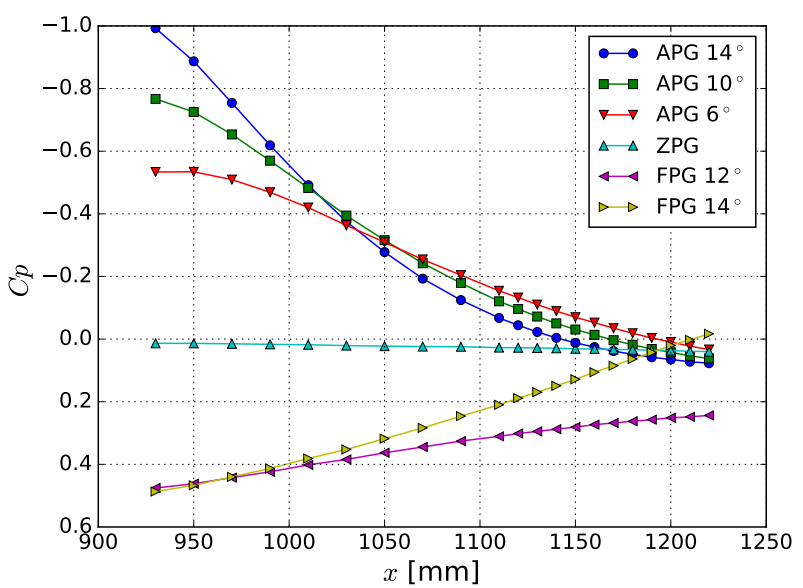

Figure 5: Cp distributions in streamwise direction between $930 \mathrm{~mm} \leq x \leq 1220 \mathrm{~mm}, U_{0}=30.2 \mathrm{~m} / \mathrm{s}$.

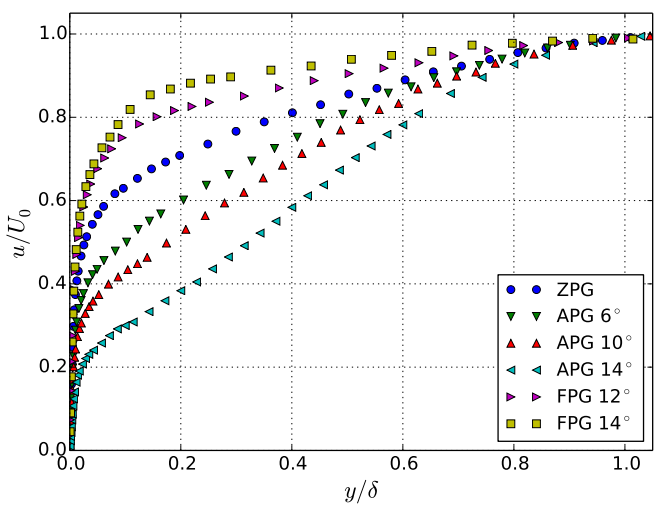

(a)

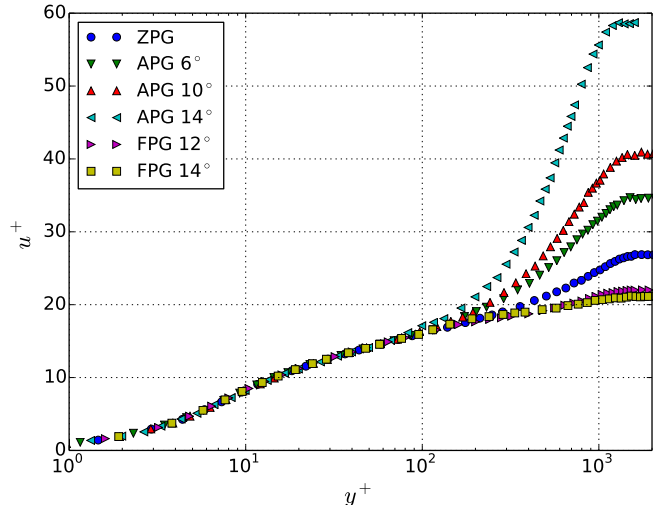

(b)

Figure 6: Mean velocity profiles at $x=1210 \mathrm{~mm}, U_{0}=30.2 \mathrm{~m} / \mathrm{s}$.

Table 2 summarizes the relevant APG and FPG boundary layer parameters for the two selected velocity measurement positions at the rear part of the plate. Note that for the APG cases the local pressure gradients $d p / d x$ and the NACA 0012 geometrical angles-of-attack show inconsistent trends, whereas consistent trends are limited to the more upstream locations (cf. Fig. 5). 
From Fig. $6(x=1210 \mathrm{~mm})$ one could conclude, that both the shape factor $H$ and Clauser's equilibrium parameter $\beta_{\delta^{*}}$ can be used to correctly represent the described ZPG and APG effects on the mean velocity profile shape. However, compared to $H, \beta_{\delta^{*}}$ is directly impacted by the local pressure gradient, and a stronger local $d p / d x$ does not necessarily indicate the upstream boundary layer developmental history of a much stronger initial APG or FPG.

For example, if we compare $\beta_{\delta^{*}}$ and $H$ as derived at the two different measurement positions for the APG $-10^{\circ}$ and $\mathrm{APG}-14^{\circ}$ cases, the values show reverse trends: when moving downstream from $x=1128 \mathrm{~mm}$ to $x=1210 \mathrm{~mm}$, the parameter $H$ increases, whereas $\beta_{\delta^{*}}$ decreases. In Fig $7(\mathrm{a})$ it is documented, that the measured velocity profiles cannot be sorted in the correct order based on $\beta_{\delta^{*}}$. On the contrary, $H$ perfectly captures the developed trends. The measured profile shapes indicate that the velocity profiles at $x=1210 \mathrm{~mm}$ are still significantly affected by the strong initial APG conditions. A plot of the corresponding defect profiles is shown in Fig $7(\mathrm{~b})$. An equilibrium boundary layer presents a larger velocity gradient in the outer layer for a greater value of $\beta_{\delta^{*}} \cdot{ }^{13-15}$ The measured APG boundary layers in the current study show reversed trends, indicating that Clauser's equilibrium parameter $\beta_{\delta *}$ is not suited to define the shape of the velocity profiles for arbitrary non-equilibrium boundary layers, especially for cases with fast pressure gradient changes.

Table 2: Boundary layer parameters for APG and FPG, $U_{0}=30.2 \mathrm{~m} / \mathrm{s}$.

\begin{tabular}{lcccccccccc}
\hline \hline & $\begin{array}{c}U_{0} \\
(\mathrm{~m} / \mathrm{s})\end{array}$ & $\begin{array}{c}\delta \\
(\mathrm{mm})\end{array}$ & $\begin{array}{c}\delta^{*} \\
(\mathrm{~mm})\end{array}$ & $\begin{array}{c}\theta \\
(\mathrm{mm})\end{array}$ & $\begin{array}{c}u_{\tau} \\
(\mathrm{m} / \mathrm{s})\end{array}$ & $R e_{\tau}$ & $R e_{\theta}$ & $\begin{array}{c}d p / d x \\
(\mathrm{~Pa} / \mathrm{m})\end{array}$ & $\beta_{\delta^{*}}$ \\
\hline$x=1128 \mathrm{~mm}$ & & & & & & & & & \\
\hline APG -14 & 31.2 & 26.3 & 7.38 & 3.87 & 1.91 & 0.645 & 1102 & 7831 & 1084 & 16.2 \\
APG -10 & 32.0 & 23.0 & 5.09 & 3.12 & 1.63 & 0.88 & 1314 & 6492 & 1225 & 6.8 \\
APG -6 & 32.3 & 20.0 & 3.88 & 2.56 & 1.52 & 1.03 & 1338 & 5362 & 1156 & 3.5 \\
FPG 12 & 26.1 & 18.1 & 2.55 & 1.92 & 1.32 & 1.12 & 1316 & 3258 & -373 & -0.6 \\
FPG 14 & 28.2 & 16.2 & 1.73 & 1.35 & 1.28 & 1.295 & 1362 & 2469 & -1060 & -0.9 \\
\hline$x=1210 \mathrm{~mm}$ & & & & & & & & & \\
\hline APG -14 & 29.9 & 35.0 & 12.07 & 5.69 & 2.12 & 0.51 & 1159 & 11046 & 320 & 12.5 \\
APG -10 & 30.4 & 28.7 & 7.68 & 4.39 & 1.75 & 0.745 & 1388 & 8670 & 518 & 6.0 \\
APG -6 & 30.8 & 24.4 & 5.61 & 3.49 & 1.61 & 0.89 & 1410 & 6979 & 643 & 3.8 \\
FPG 12 & 27.2 & 15.9 & 1.96 & 1.52 & 1.29 & 1.235 & 1275 & 2683 & -209 & -0.2 \\
FPG 14 & 31.1 & 13.8 & 1.28 & 1.01 & 1.26 & 1.47 & 1317 & 2040 & -1006 & -0.5 \\
\hline \hline
\end{tabular}

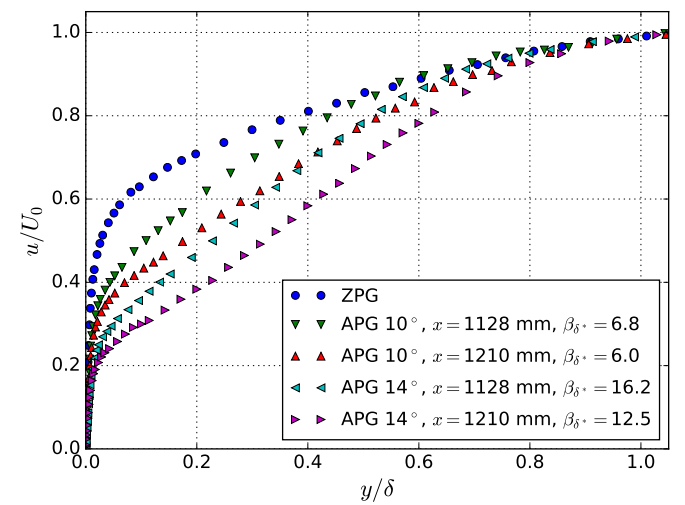

(a)

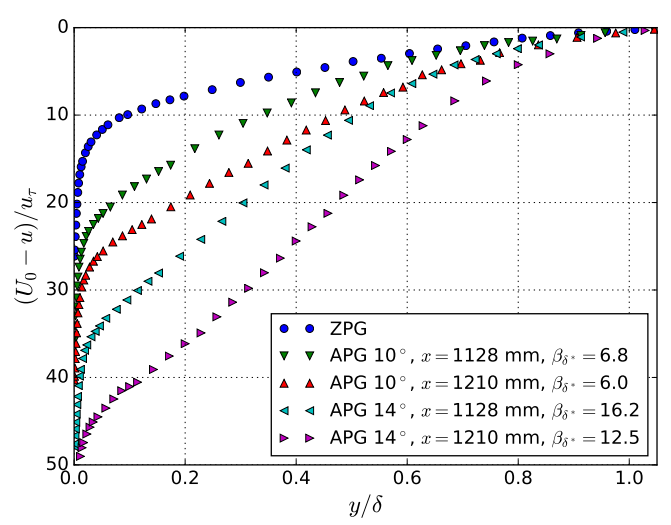

(b)

Figure 7: Mean velocity profiles for ZPG and APG $-10^{\circ}$ and $-14^{\circ}, U_{0}=30.2 \mathrm{~m} / \mathrm{s}$.

\section{III.B. One-point spectra}

Fig. 8(a) shows the surface pressure one-point power spectral densities (psd) for ZPG boundary layers at $x=1210 \mathrm{~mm}$. Spectral levels in this paper are referenced to a $20 \mu \mathrm{Pa}$ reference pressure. The spectra show an overall good agreement with predictions applying Goody's ${ }^{6}$ model. Especially for the higher 
velocities the measured spectra are contaminated by setup-related disturbances at both low and high frequencies; the spectral increase at frequencies below about $230 \mathrm{~Hz}$ for $U_{0}=58.7 \mathrm{~m} / \mathrm{s}$ is due to the impact of the open-jet free shear layer (featuring a much higher velocity scaling exponent than the turbulent boundary-layer wall pressures) while the resonance frequency for the Kulite-pinhole-arrangement was located at about $30 \mathrm{kHz}$. However, an impact of the resonance is found down to $7 \mathrm{kHz}$ for $49.1 \mathrm{~m} / \mathrm{s}$ and $58.7 \mathrm{~m} / \mathrm{s}$. Spectral levels below $48 \mathrm{~dB}$ are buried by the electrical noise of the applied Kulite sensor.

Remaining minor deviations are observed with regard to the maximum location and the low-frequency slopes at the lower velocities.

When scaled based on mixed variables, as Goody proposes, the normalized spectra collapse to a single curve at mid Strouhal numbers $\omega \delta / U_{e}$ with a slope of $\omega^{-0.7}$, see Fig. 8(b). The turbulent boundary layer edge velocity $U_{e}$ is herein set to $U_{e}=0.99 U_{0}$. This mid Strouhal number range is primarily attributed to the log-law region of the boundary layer at a ZPG. ${ }^{1}$ The similarity of the velocity profile might predominantly contribute to the collapse of the spectra at these mid frequencies. A Reynolds number effect at high frequencies is expressed as an elongated mid frequency $\omega^{-0.7}$ range, which is well identified by Goody using the model parameter $R_{T}=R e_{\tau} \sqrt{C_{f} / 2}$, where $C_{f}$ is the skin friction coefficient. Different trends are found at low Strouhal numbers, where the measured spectra appear free of disturbances: the present measurements indicate also a dependence of the low-frequency slope on Reynolds number, whereas Goody proposes identical spectral shapes in the low Strouhal number range. Particularly, at $U_{0}=20.3 \mathrm{~m} / \mathrm{s}$ the spectral increase is steeper at low frequencies and the maximum is located at a higher Strouhal number compared to the spectra for the other velocities. Similar observations were made by Farabee and Casarella ${ }^{5}$ and Leclerco. ${ }^{22}$ Panton \& Linebarger $^{23}$ and $\mathrm{Hu}$ et al. ${ }^{24,25}$ calculated the contributions from different decks of the boundary layer and found that the spectra at low frequencies are composed of contributions from both the inner and outer layers. Based on this argument it is reasonable to conclude that an elongated mid frequency range might change the shape of the spectra also at low frequencies. Note that the slightly changed velocity profile shape at smaller $U_{0}$, also discussed as a Reynolds number effect, is in line with the observation of a changed shape of the corresponding surface pressure spectrum.

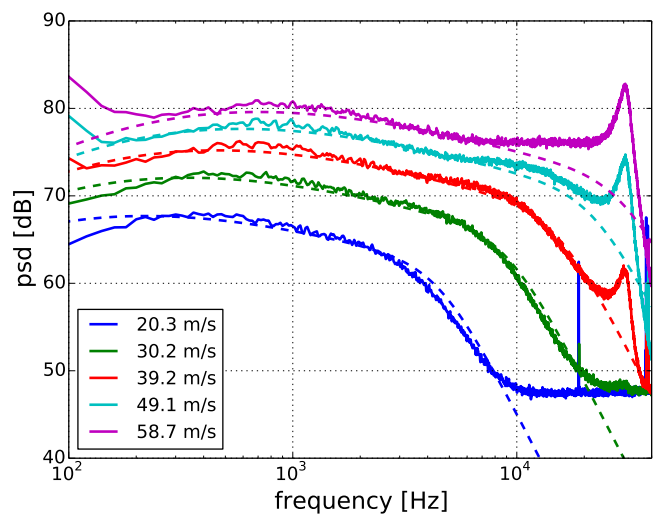

(a)

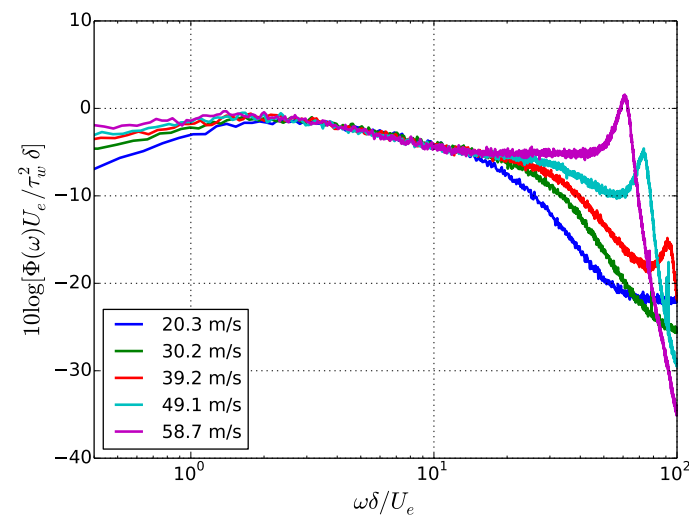

(b)

Figure 8: (a) One-point spectra for different velocities compared with predictions according to Goody, ${ }^{6}$ $x=1210 \mathrm{~mm}$; (-), measured spectra; (- -), Goody spectra; (b) spectra scaled by $\tau_{w}$ as pressure scale and $\delta / U_{e}$ as time scale.

Figs. 9(a-e) show the surface pressure one-point spectra for APG and FPG boundary layers at $U_{0}=30.2 \mathrm{~m} / \mathrm{s}$. The corresponding ZPG spectrum is plotted as reference. The formation of the spectra in dependence of the initial pressure gradient conditions is well illustrated. Compared to the ZPG spectra the APG spectra feature an increase in maximum level and a steeper slope at mid frequencies with consistent trends as observed for the corresponding velocity profiles. For a given initial APG configuration, when moving downstream, the spectra shift towards lower frequencies and the slope at mid frequencies becomes successively steeper. For the FPG configurations spectral energy is decreased at mid frequencies and the slope in this range is successively flattened, when moving downstream. A strong interference occurs at low frequencies for the FPG $14^{\circ}$ case, which is probably caused by the fully separated flow on the suction side of the NACA airfoil.

Fig. 9(f) shows the scaled spectra for ZPG, APG and FPG boundary layers at $x=1210 \mathrm{~mm}$ using the same scaling variables as for the ZPG spectra. Unlike the good collapse of the ZPG spectra, normalized levels diverge by up to $20 \mathrm{~dB}$. Again, the evolution of the mid frequency slope from a FPG boundary 


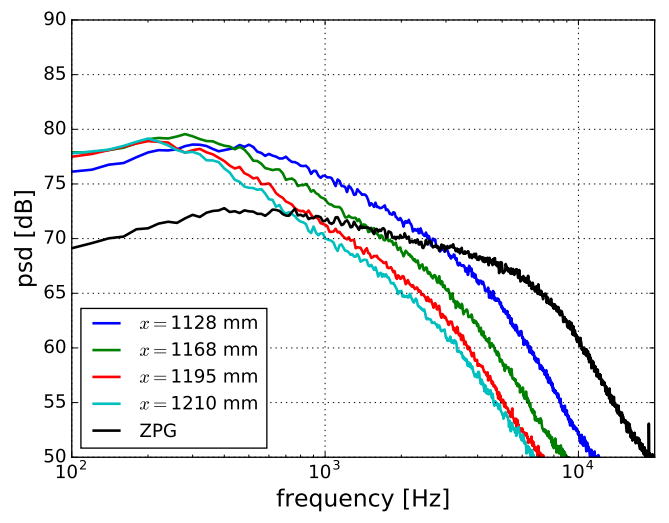

(a)

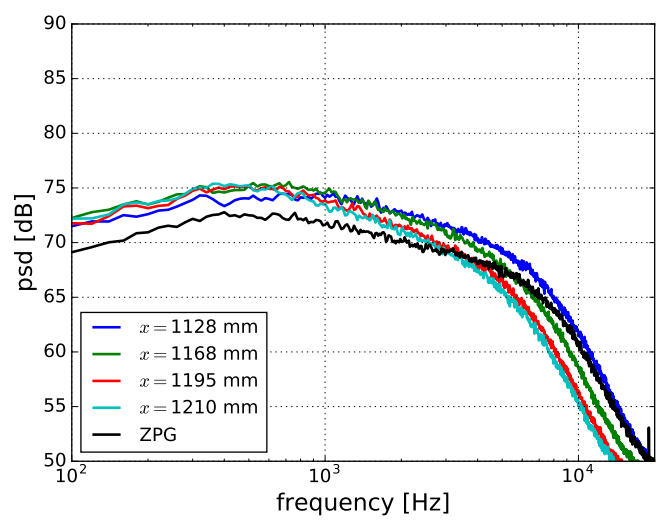

(c)

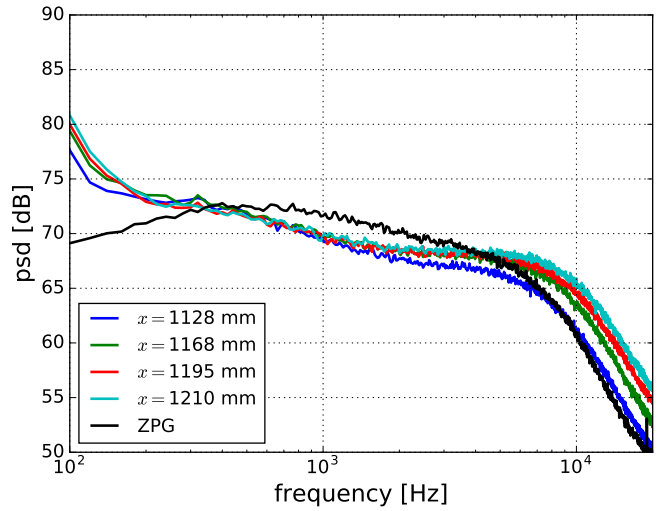

(e)

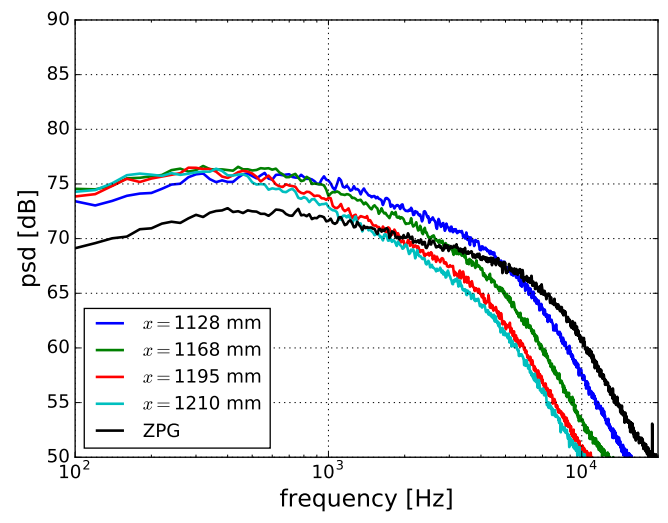

(b)

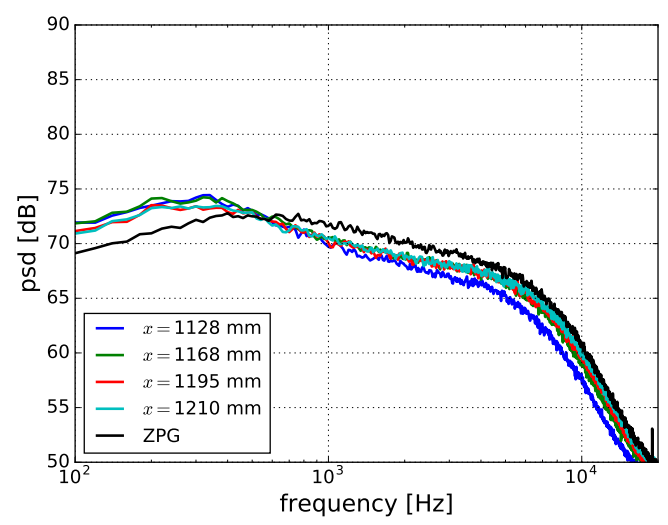

(d)

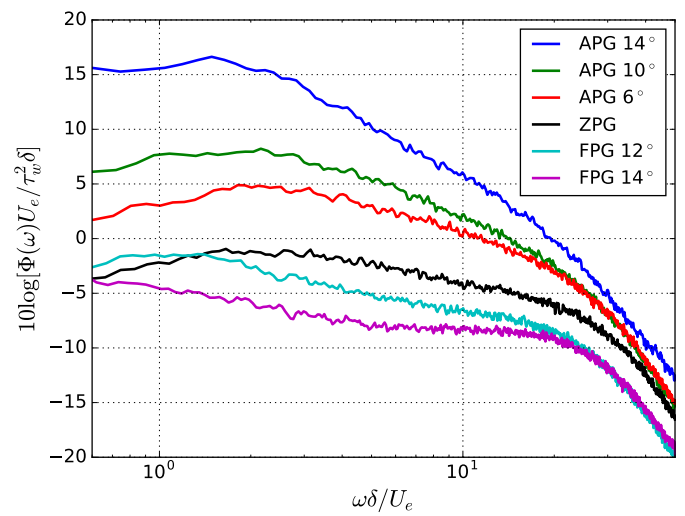

(f)

Figure 9: One-point spectra for $U_{0}=30.2 \mathrm{~m} / \mathrm{s}$; (a) APG - $14^{\circ}$; (b) APG - $10^{\circ}$; (c) APG - $6^{\circ}$; (d) FPG $12^{\circ}$; (e) FPG $14^{\circ}$; (f) spectra at $x=1210 \mathrm{~mm}$, scaled by $\tau_{w}$ as pressure scale and $\delta / U_{e}$ as time scale.

layer to an APG boundary layer is well illustrated. Note that the roll-off slope of $\omega^{-5}$ at high frequencies appears to be unaffected for both APG and FPG spectra. A fairly good collapse for the ZPG and APG spectral maxima is found by scaling with $u_{\tau} / Q^{2} \theta$ and $\omega \theta / U_{0}$, see Fig. 10(a). All the measured APG spectra at $x=1128 \mathrm{~mm}$ and $1210 \mathrm{~mm}$ including the freestream velocities of $20.3 \mathrm{~m} / \mathrm{s}$ and $39.1 \mathrm{~m} / \mathrm{s}$ are scaled using these variables and a good collapse of the spectral maxima is shown in Fig. 10(b). It is worth to note that a comparably good collapse is also found for spectral scaling based on $u_{\tau} / Q^{2} \delta$ or $U_{0} / Q^{2} \delta^{*}$. All the scalings are based on the dynamic pressure $Q$. It seems to be more reasonable to scale the APG spectra using the outer pressure scale $Q$ instead of $\tau_{w}$. However, other than the commonly used outer variables $U_{0} / Q^{2} \delta$, a mixed representation for the involved time scales is used to consider the 


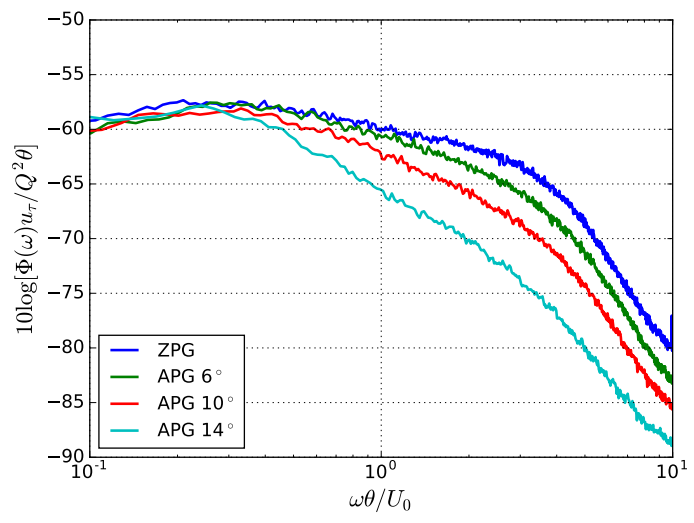

(a)

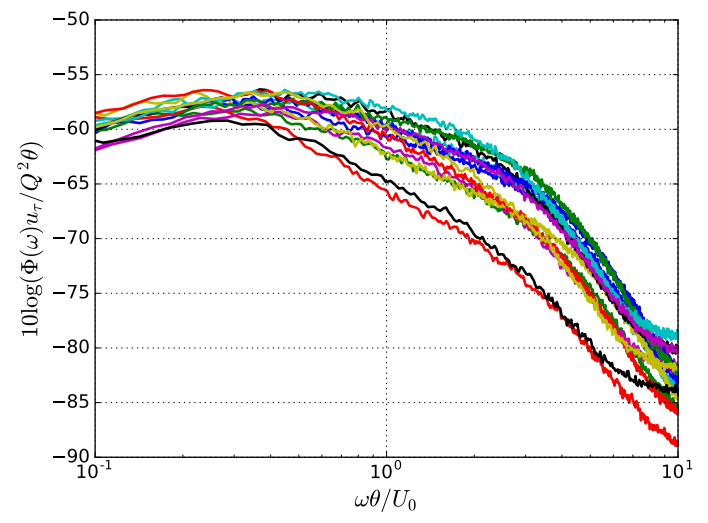

(b)

Figure 10: Spectra scaled by $u_{\tau} / Q^{2} \theta$ and $\theta / U_{0}$; (a) spectra for ZPG and APG at $x=1210 \mathrm{~mm}$ for the freestream velocity of $30.2 \mathrm{~m} / \mathrm{s}$; (b) spectra for APG.

effect of an APG boundary layer.

\section{III.C. Spectral model for APG wall pressure fluctuations}

From the analysis of the one-point spectra, we found a fairly good collapse of the spectral maxima when using the outer variables; a clear trend for the slope at mid frequencies and a nearly unchanged high frequency roll-off slope was observed. From these observations we are encouraged to develop an empirical spectral model for APG wall pressure fluctuations. A new empirical model based on the present measured data is proposed in this section.

To some extent, the APG spectra own similar trends as the ZPG spectra. The spectra increase first at low frequencies, then drop at mid frequencies and roll off at high frequencies. Goody's model can represent these trends for ZPG spectra for the three different ranges well, especially at mid- and high frequencies. From this point it is appropriate to take Goody's model as the starting point. Goody's model $^{6}$ is expressed as

$$
\frac{\Phi(\omega) U_{e}}{\tau_{w}^{2} \delta}=\frac{a \cdot\left(\omega \delta / U_{e}\right)^{b}}{\left[\left(\omega \delta / U_{e}\right)^{c}+d\right]^{e}\left[f \cdot\left(\omega \delta / U_{e}\right)\right]^{g}},
$$

where the value of the variables $a-g$ was obtained by fitting the measurement data from literature, $a=3$, $b=2, c=0.75, d=0.5, e=3.7, f=1.1 R_{T}^{-0.57}$ and $g=7$. Goody used $U_{e} / \tau_{w}^{2} \delta$ and $\omega \delta / U_{e}$ as scaling variables for the ZPG spectra. However, based on the previous discussion is more appropriate to use $u_{\tau} / Q^{2} \theta$ and $\omega \theta / U_{0}$ as the scaling variables for the APG spectra. It is worth to mention that the chosen scaling variables provide a better trend in fitting the maximum locations of the spectra than the other two proper scaling variables mentioned before based on the measured data. Thus, Goody's model is rewritten as

$$
\frac{\Phi(\omega) u_{\tau}}{Q^{2} \theta}=\frac{a \cdot\left(\omega \theta / U_{0}\right)^{b}}{\left[\left(\omega \theta / U_{0}\right)^{c}+d\right]^{e}\left[f \cdot\left(\omega \theta / U_{0}\right)\right]^{g}} .
$$

The variables $a_{-} g$ in this equation control the shape of the dimensionless spectra. The amplitude of the spectra is adjusted by the value of $a$. The slopes in different frequency ranges are driven by the combination of $b, c, e$ and $g$. The variable $f$ determines the extension of the mid frequency range. The maximum location is affected by the value of $d$, the slope at low frequencies and the trend of the transition range between the increase and decrease at low and mid frequencies.

The first step of the modification is to represent the ZPG spectra which are supposed to have a trend of $\omega^{-0.7}$ at mid frequencies and $\omega^{-5}$ at high frequencies. This trend can be realized by means of the combination of the variables, which follows $b-c \cdot e=-0.7$ and $b-g=-5$. Goody adapted $b=2$ from the Chase-Howe ${ }^{26}$ model, which implies a $\omega^{2}$ increase at low frequencies. Panton \& Linebarger ${ }^{23}$ and Blake ${ }^{1}$ calculated the spectra of the wall pressure fluctuations by solving a Poisson equation. The result is derived by integral of the contributions throughout all decks of the boundary layer. Due to the term $k_{1}^{2} / k^{2} \exp (-2 k y)$ in the equation, where $k_{1}$ is the wavenumber in longitudinal direction, $k^{2}=k_{1}^{2}+k_{3}^{2}$ and $y$ is the wall-normal distance to the wall, an $\omega^{2}$ increase at low frequencies is obtained. However, in the calculation the flow is assumed as frozen turbulence. Hu et al. ${ }^{24,25}$ calculated the spectra for both frozen turbulence and non-frozen turbulence. The result showed that the $\omega^{2}$ increase 
does not hold if a non-frozen turbulence is considered. The effect of turbulence decay is only noticeable at higher frequencies for the velocity spectra. However, the wall pressure spectra are also affected at lower frequencies due to the extra term $k_{1}^{2} / k^{2} \exp (-2 k y)$. As a result, the slope of the low-frequency increase turns out to be smaller. Furthermore, in the Poisson equation the mean-shear turbulence interaction term is considered as the dominant source term, thus, the turbulence-turbulence interaction term is dropped in the calculation. Kraichnan ${ }^{27}$ and Meecham \& Tavis $^{28}$ calculated the importance of the mean-shear term and demonstrated the dominance of the mean-shear term for the wall mean square pressure. The simulation results of $\mathrm{Hu}^{24,25}$ showed that the mean-shear term dominates the contribution to wall pressure fluctuations at mid and high frequencies, however, the turbulence-turbulence term, with an almost plateau-like spectrum at low frequencies, gains importance and becomes comparable to the mean-shear term at very low frequencies. A similar statement was obtained theoretically by $\operatorname{Hodgson}^{29}$ for the wall pressure spectra on a glider wing. Thus, the slope in the low frequency range could be also affected by considering the effect of the turbulence-turbulence term. A precise measurement at very low frequencies is difficult mostly due to the limitation of the experimental facilities, e.g. high background noise level at low frequencies. Until now only Farabee \& Casarella ${ }^{5}$ measured the $\omega^{2}$ increase at the lowest frequency range $<10 \mathrm{~Hz}\left(\omega \delta / U_{0}<0.08\right)$ by means of noise cancelation technique. At low frequencies their results showed an approx. $\omega^{0.3}$ increase. In the literature an increase between $\omega^{0.2-0.8}$ at low frequencies is found for the ZPG spectra.

The APG spectra in the present experiment show $\omega^{0.6-1.0}$ at low frequencies. A steeper slope was found in experiments from Catlett et al. ${ }^{10}$ and Suryadi \& Herr. ${ }^{12}$ The results showed a larger low frequency increase at a greater APG and the increase can reach about $\omega^{1.4}$ as the APG boundary layer approaches separation. From those observations it can be drawn that the low frequency slope for an APG spectra is strongly dependent on the velocity profile of the boundary layer and probably also affected by the Reynolds number. However, for the present measured data, due to the low frequency contamination from the free shear layer, it is not possible to figure out the dependence between the low frequency slope and the possible important parameters. Thus, in this work a constant $b=1.0$ is applied which is considered as an averaged value for the APG spectra. Since the value of $b$ is fixed, we can determine $g=b+5=6.0$ and $c \cdot e=b+0.7=1.7$. An additional variable $h$ is added to govern the slope at mid frequencies, which works as a combination with $c$ and $e$ and prescribe the slope as $c \cdot e \cdot h-b$, e.g. a larger $h$ leads a steeper decrease at mid frequencies. It is noted that a steeper decrease at mid frequencies follows a more rapid transition between the increase at low frequencies and the decrease at mid frequencies. This feature requires an increasing value of $c$ as the decrease slope at mid frequencies steepens, because the slope of the transition range is primarily managed by $\left(\omega \theta / U_{0}\right)^{c}$ in Eq. 3. Finally, to determine the value of $c$ and $e$, and the proper way to introduce $h$, the value and the combination of those variables should be able to characterize the transition range for both small and large decrease slopes at mid frequencies.

Fig. 11 shows the perfect fittings for the measured smallest and largest decrease slope using the

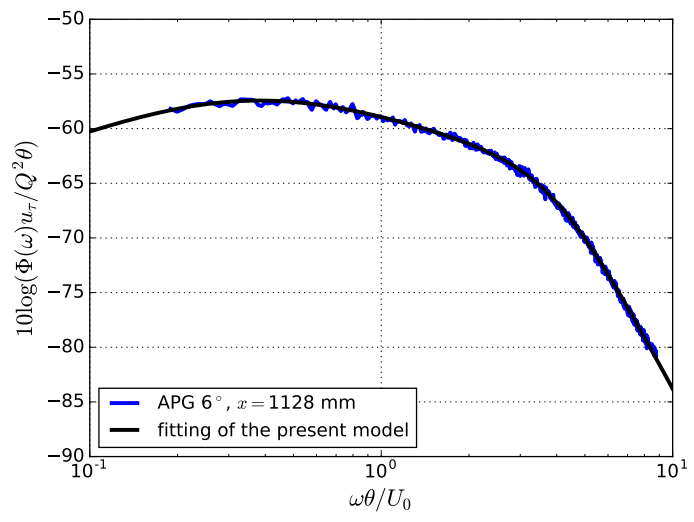

(a)

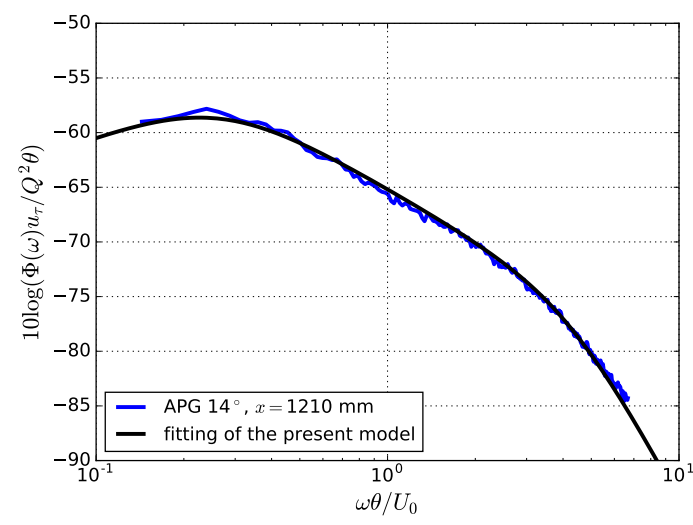

(b)

Figure 11: Comparison between the measurement data and the fitting of the present model using the selected value of the variables; (a) APG $-6^{\circ}$ at $x=1128 \mathrm{~mm}$; (b) APG $-14^{\circ}$ at $x=1210 \mathrm{~mm}$. 
following combination expressed as

$$
\frac{\Phi(\omega) u_{\tau}}{Q^{2} \theta}=\frac{a \cdot\left(\omega \theta / U_{0}\right)^{1.0}}{\left[\left(\omega \theta / U_{0}\right)^{1.5 \cdot h^{1.6}}+d\right]^{1.13 / h^{0.6}}\left[f \cdot\left(\omega \theta / U_{0}\right)\right]^{6.0}},
$$

where the variables are determined as follows: $c=1.5$ and $e=1.13$. It is found that the change of the transition slope is too fast to be catched by a single factor $h$ and a factor of $h^{1.6}$ can fairly well identify the transition slope. A $h^{0.6}$ is introduced as $1.13 / h^{0.6}$ to hold the concept for the combination of $c \cdot e \cdot h$. Consequently, four variables remain to be determined and this is done by fitting all measured APG spectra. The task now is to find some dependence between the variables and the potential determining boundary layer parameters. The considerations are as follows: 1, the amplitude manager $a$ depends on the variable $d .2$, the mid frequency slope controller $h$ is directly impacted by the boundary layer velocity profile. From the previous discussion, the shape factor $H$ is a proper choice as it directly correlates to the velocity profile. 3 , the variable $d$ impacts the maximum location of the spectra. It is considered that it could be dependent on both the velocity profile and the Reynolds number. During the test it is found that the combination of $R e_{\theta} H$ and $R e_{\theta} /\left(\delta / \delta^{*}\right)$ show good results. To keep the model as simple as possible by avoiding to introduce new parameters, $R e_{\theta} H$ is chosen. 4 , the variable $f$ determines the extension of the mid frequency decrease range and should depend on $R e_{\tau}$. Goody used $R e_{T}=R e_{\tau} \sqrt{C_{f} / 2}$ to prescribe the extension and good agreement to the experimental results is shown. However, for the applied time scale variables $\omega \theta / U_{0}, R e_{\tau}$ shows a better agreement to the results. Fig. 12 shows the obtained value for the variables by fitting the measured wall pressure spectra against the selected parameters. Thus, the variables as a function of the boundary layer parameters can be determined as follows:

$$
\begin{aligned}
a & =(81.004 d+2.154) \cdot 10^{-7} \\
d & =10^{-5.8 \cdot 10^{-5} \operatorname{Re}_{\theta} H-0.35} \\
h & =1.169 \ln (H)+0.642 \\
f & =7.645 R e_{\tau}^{-0.411}
\end{aligned}
$$

The curves of the resulting functions match fairly well with the obtained value of the variables, see Fig 12. This indicates that the selected boundary layer parameters can characterize the features of the APG spectra.

Fig 13 shows the prediction of the present model, RRM model ${ }^{9}$ and CFAS model. ${ }^{11}$ RRM model and CFAS model both modified the variables $a^{-} g$ of Goody's model as functions of the boundary layer parameters. The key differences between the present model and RRM model \& CFAS model are as follows: 1 , the present model uses a more representative normalization with $u_{\tau} / Q^{2} \theta$ for the APG spectra instead of $U_{e} / \tau_{w}^{2} \delta^{*}$ uesd in RRM model or $U_{e} / \tau_{w}^{2} \delta$ in CFAS model. 2, the present model uses the shape factor $H$ to operate on the mid frequency decrease slope. Contrarily, RRM model and CFAS model used the Clauser's equilibrium parameter as the driving parameter, which based on the former discussion is not necessarily appropriate for applying in an arbitrary APG boundary layer. 3, the variable $b$ which manages the low frequency increase slope is changed to $b=1.0$ in the present model. In RRM model and CFAS model $b=2$ adapted from Goody's model.

A very good prediction is obtained using the present model compared to the present measured spectra, see Fig 13(a-b). However, RRM model shows no clear decrease slope at mid frequencies and CFAS model fails in both amplitudes and shapes. A comparison between the three models to the results from Schloemer ${ }^{8}$ and Catlett et al. ${ }^{10}$ is shown in Fig $13(\mathrm{c}-\mathrm{d})$. The boundary layer parameters for the calculation case are listed in Table 3. The present model shows fairly good prediction of Schloemer's result both amplitude and the spectral trend at lower frequencies. However, the measured spectrum rolls off at higher frequencies. Schloemer measured the spectra using a $1.5 \mathrm{~mm}$ diameter flush-mounted sensor and corrected the spectra with $\operatorname{Corcos}^{30}$ correction, which takes the importance of the turbulence decay and the convection feature on a finite sensor size into account. The authors can not evaluate up to how large dimensionless frequency works the Corcos correction well. However, the Corcos correction was obtained on the basis of the longitudinal- and lateral turbulence decay in a ZPG boundary. The present data and the measured data from Schloemer show a more rapid longitudinal turbulence decay in an APG boundary layer and a faster decay causes a larger attenuation for a same sized sensor. Thus, an attenuation on Schloemer's spectra at higher frequencies is possible. The CFAS model shows a similar slope as the present model, however, a much lower amplitude compared to the measured spectrum. Fig 13(d) shows the comparison to the results from Catlett et al. ${ }^{10}$ The present model shows a perfect match at mid and high frequencies. However, due to a lower predicted maximum location the maximum amplitude is about $5 \mathrm{~dB}$ larger than the measured spectrum. Note that the $R_{\theta}$ in that measurement is very large and is not covered by the Reynolds number range in the present measurement. It is considered that 


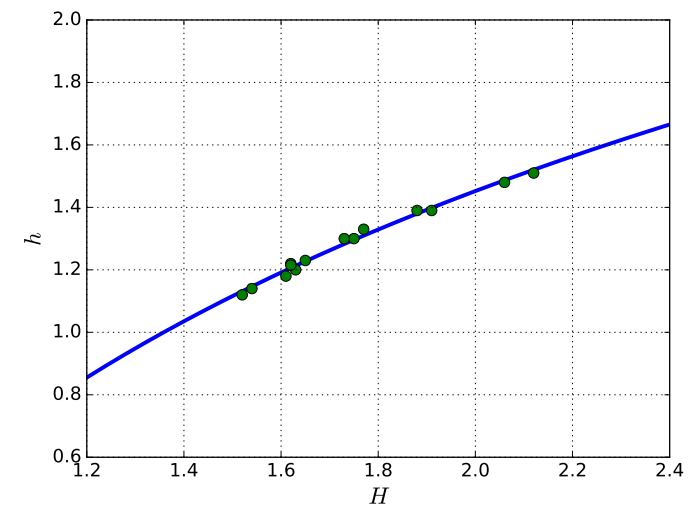

(a)

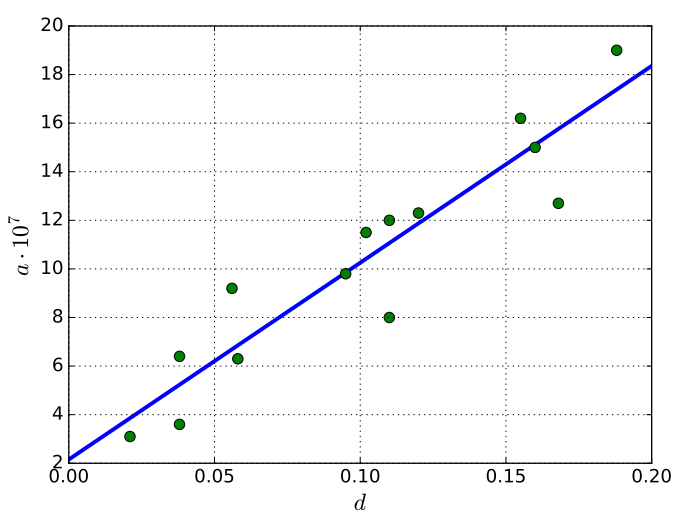

(c)

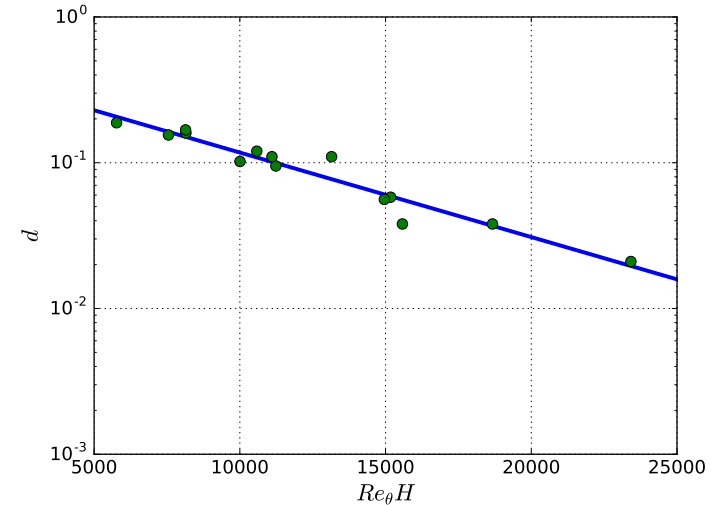

(b)

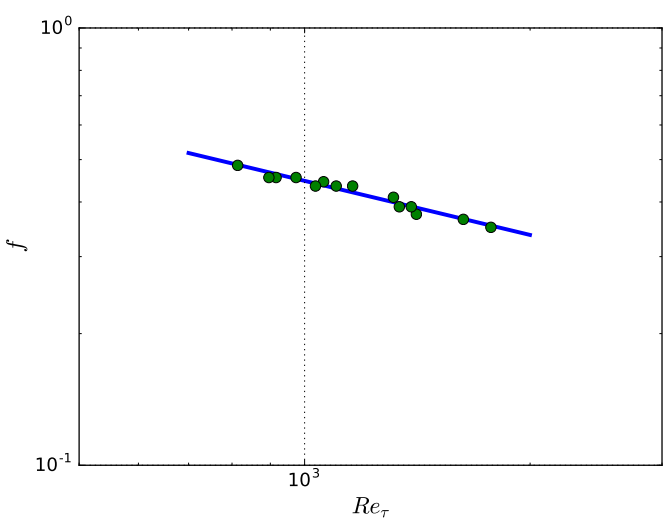

(d)

Figure 12: Obtained value by fitting the APG spectra and the resulting functions for the variables.

the Reynolds number effect may be minimized at very large Reynolds number, otherwise the maximum location will be continually shifted to lower frequencies as the Reynolds number increases, which may be not physically realistic. Again, the RRM model rolls off at mid frequencies and fails to predict the slope at higher frequencies. Both RRM and CFAS models predict a steeper slope at low frequencies compared to the measured spectrum due to the applied value $b=2$ in the models.

Table 3: Boundary layer parameters for Schloemer's and Catlett's experiments

\begin{tabular}{ccccccccccc}
\hline \hline & $\begin{array}{c}U_{0} \\
(\mathrm{~m} / \mathrm{s})\end{array}$ & $\begin{array}{c}\delta \\
(\mathrm{mm})\end{array}$ & $\begin{array}{c}\delta^{*} \\
(\mathrm{~mm})\end{array}$ & $\begin{array}{c}\theta \\
(\mathrm{mm})\end{array}$ & $\begin{array}{c}u_{\tau} \\
(\mathrm{m} / \mathrm{s})\end{array}$ & $R e_{\tau}$ & $R e_{\theta}$ & $\begin{array}{c}d p / d x \\
(\mathrm{~Pa} / \mathrm{m})\end{array}$ & $\beta_{\delta^{*}}$ \\
\hline Schloemer & 43.6 & 25.6 & 5.26 & 3.33 & 1.58 & 1.29 & 2144 & 9428 & 1237 & 3.27 \\
Catlett et al. $^{*}$ & 26.0 & 80.1 & 24.3 & 12.0 & 2.03 & 0.40 & 2081 & 20260 & 680 & 87.0 \\
\hline \hline
\end{tabular}

*The value of $d p / d x$ is estimated by gaining the best match for CFAS model fitting the measured data because the value is not available for the selected measurement point.

\section{III.D. Cross-spectra and convection features}

From the two-point statistics obtained from the sensors located in different longitudinal- and lateral positions, the spatial and temporal properties of the wall fluctuating pressure field can be studied. $\operatorname{Corcos}^{7}$ used exponential functions to characterize the longitudinal- and lateral coherence of the pressure field by taking advantage of the similarity of the turbulence decay, expressed as

$$
\left|\Gamma\left(r_{1}, r_{3}, \omega\right)\right|=\exp \left(-\alpha \omega r_{1} / U_{c}\right) \exp \left(-\beta \omega r_{3} / U_{c}\right)
$$




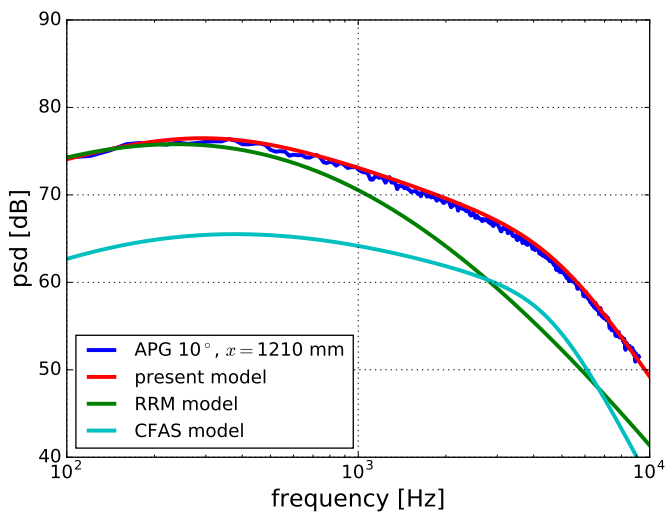

(a)

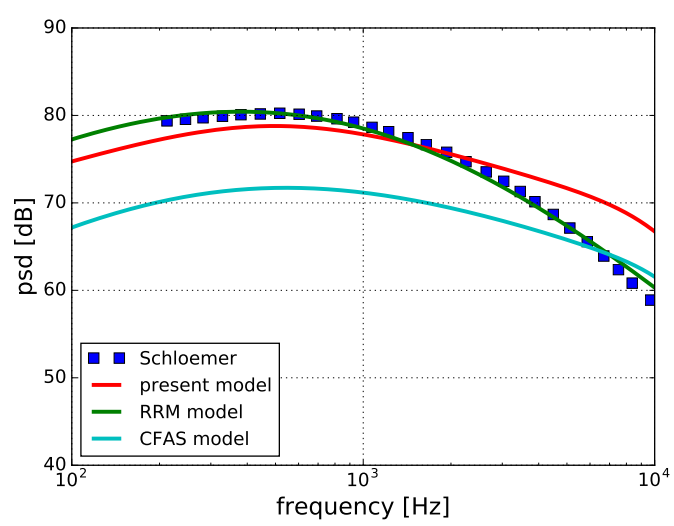

(c)

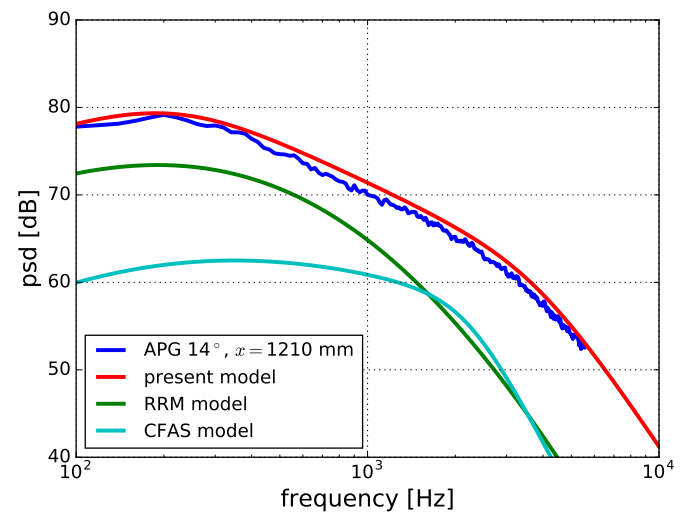

(b)

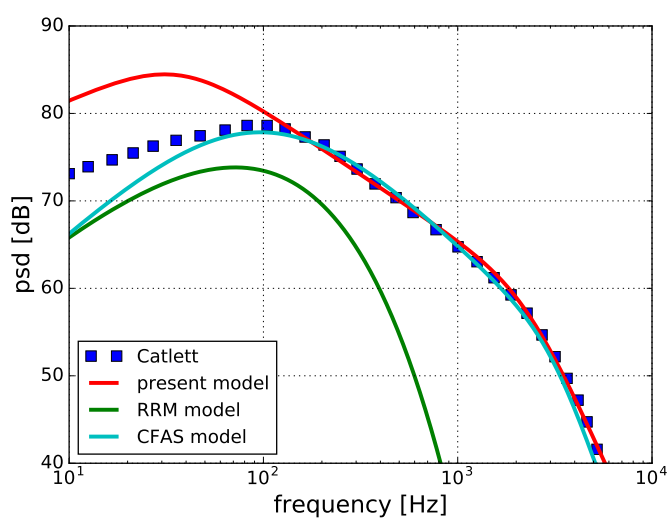

(d)

Figure 13: Prediction using the present model, RRM model and CFAS model; (a) present measurement, $\mathrm{APG}-10^{\circ}$ at $x=1210 \mathrm{~mm}$; (b) present measurement, $\mathrm{APG}-14^{\circ}$ at $x=1210 \mathrm{~mm}$; (c) Schloemer's result for $U_{0}=43.6 \mathrm{~m} / \mathrm{s}$; (d) results from Catlett et al. for $12^{\circ}$ at $U_{0}=25.6 \mathrm{~m} / \mathrm{s}$ at the most downstream measurement point.

where $\alpha$ and $\beta$ are empirical constants which are in charge of prescribing the turbulence decay in longitudinal- and lateral direction, respectively. The value of $\alpha$ depends on the Reynolds number. Generally, a smaller value relates to a larger Reynolds number. In the present measurement for ZPG boundary layers the obtained value of $\alpha$ decreases from 0.17 to 0.125 as the Reynolds number $R e_{\tau}$ increases from 1040 to 2499. A similar trend was reported by Farabee \& Casarella, ${ }^{5}$ with $\alpha=0.145$ for $R e_{\tau}=1169$ and $\alpha=0.125$ for $R e_{\tau}=2010$. The obtained value of $\beta$ shows no noticeable dependence on Reynolds number, $\beta=0.72$ for all measured velocities in ZPG boundary layers. The value of $\beta$ is reported only in few experiments. Bull ${ }^{3}$ obtained $\beta=0.715$ in a ZPG boundary layer, which is consistent with the present results.

In Eq. 9, $U_{c}$ is the convective phase velocity of the wall fluctuating pressure field and it is defined by $U_{c}\left(r_{1}, \omega\right)=r_{1} \omega / \theta\left(r_{1}, \omega\right)$, where $\theta\left(r_{1}, \omega\right)$ is the phase difference of $\Gamma\left(r_{1}, 0, \omega\right)$. The phase velocity depends on the longitudinal separations. To present the coherence with a separation of $r_{1}$ in longitudinal direction, a phase velocity $U_{c}\left(r_{1}, \omega\right)$ obtained at the same longitudinal separation is used. However, the application for the phase velocity $U_{c}$ in the lateral direction is not explicit. Bull ${ }^{3}$ used an averaged $U_{c}(\omega)$ obtained from the longitudinal direction. Brooks \& Hodgson ${ }^{31}$ applied a longitudinal separation dependent $U_{c}\left(r_{1}, \omega\right)$, which used $r_{1}=r_{3}$ to calculate the lateral coherence at a separation of $r_{3}$. It is the authors' understanding that a phase velocity $U_{c}\left(r_{1} \rightarrow 0, \omega\right)$ which indicates the local phase velocity is more meaningful to apply. However, $U_{c}\left(r_{1} \rightarrow 0, \omega\right)$ can not be measured. In this work the phase velocity $U_{c}(\omega)$ obtained from the two closest sensors $r_{1}=2 \mathrm{~mm}$ at the most downstream direction is used to represent the lateral coherence.

Figs. 14-15 show the longitudinal- and lateral coherence at the freestream velocity of $30.2 \mathrm{~m} / \mathrm{s}$ for ZPG, APG and FPG boundary layers, respectively. The longitudinal coherence for APG and FPG boundary layers are only calculated from the sensors located between $1183 \mathrm{~mm}<x<1210$ mm where 


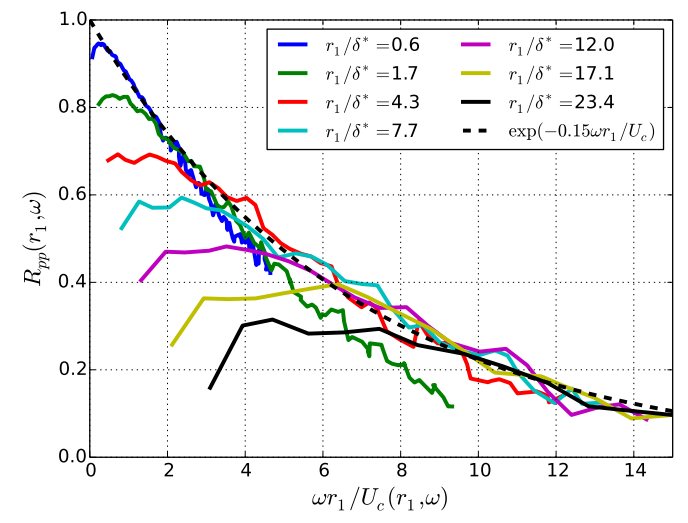

(a)

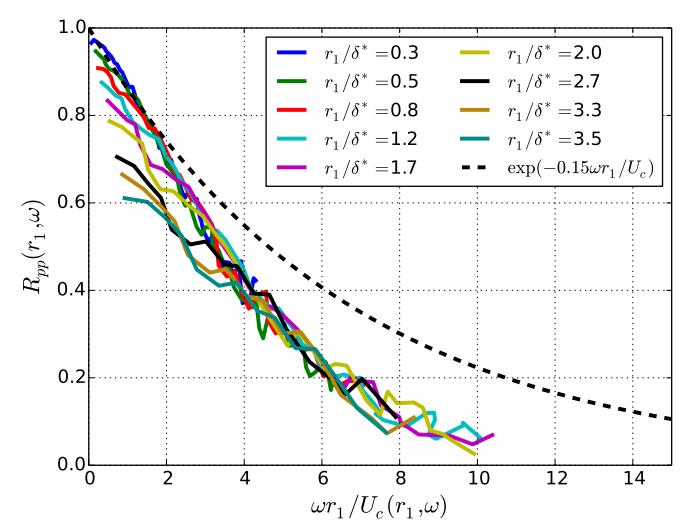

(c)

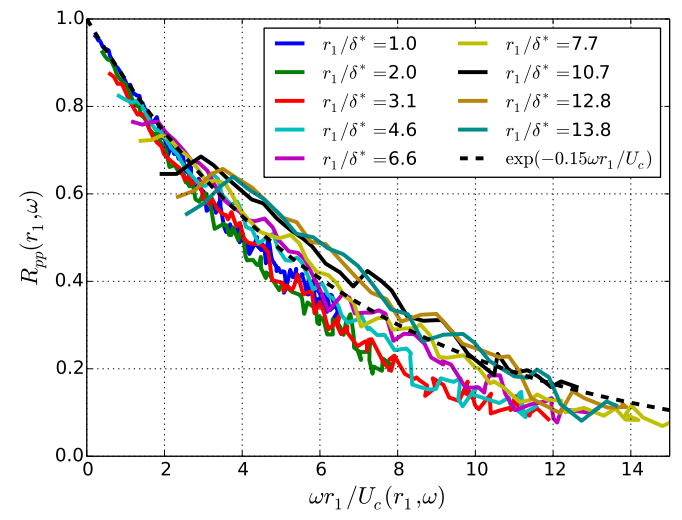

(e)

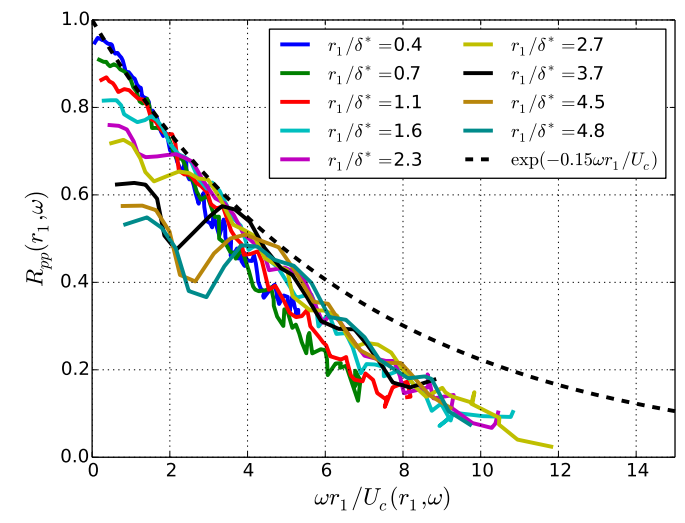

(b)

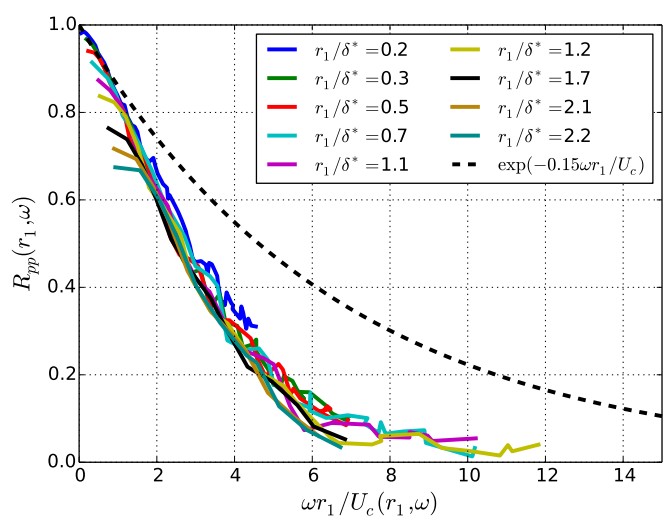

(d)

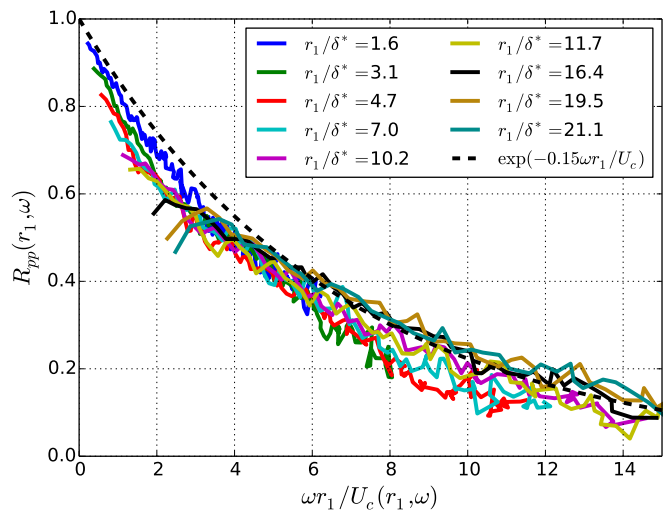

(f)

Figure 14: Longitudinal coherence as a function of $\omega r_{1} / U_{c}\left(r_{1}, \omega\right)$ at the freestream velocity of $30.2 \mathrm{~m} / \mathrm{s}$; (a) ZPG; (b) APG -6 ${ }^{\circ}$; (c) APG - $10^{\circ}$; (d) APG - $14^{\circ}$; (e) FPG $12^{\circ}$; (f) $\mathrm{FPG} 14^{\circ}$.

very similar spectra are measured. This implies a similar boundary layer condition within this range. The lateral coherence was measured at $x=1210 \mathrm{~mm}$. The longitudinal- and lateral coherence curves for the ZPG boundary layer collapse at higher frequencies and can be well fitted by the exponential functions with $\alpha=0.15$ and $\beta=0.72$, see Figs. 14(a)-15(a). The obtained exponential function for prescribing the longitudinal- and lateral coherence of the ZPG boundary layer is also plotted in the results for APG and FPG boundary layers for comparison.

Figs. 14(b-d) show the longitudinal coherence for APG boundary layers. It clearly illustrates an APG boundary layer causes an increased coherence decay in longitudinal direction, the larger the APG the stronger the decay. This implies the APG enhances the turbulence decay rate during the eddies 


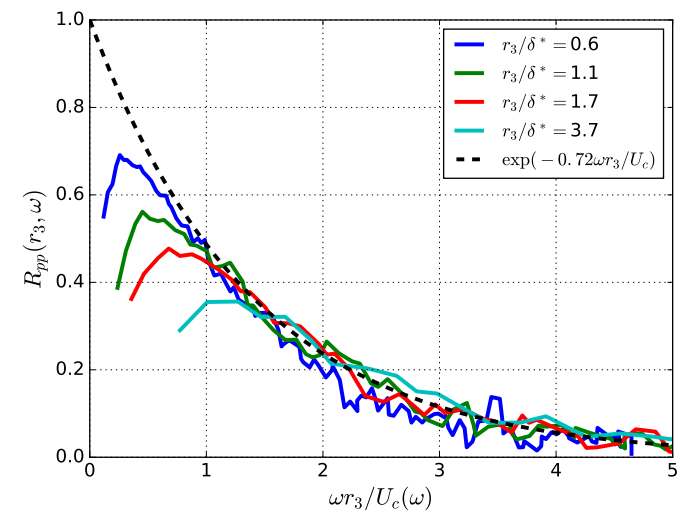

(a)

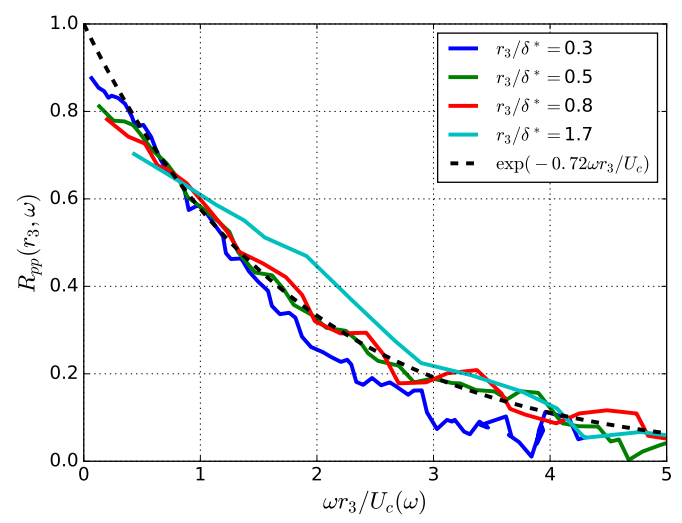

(c)

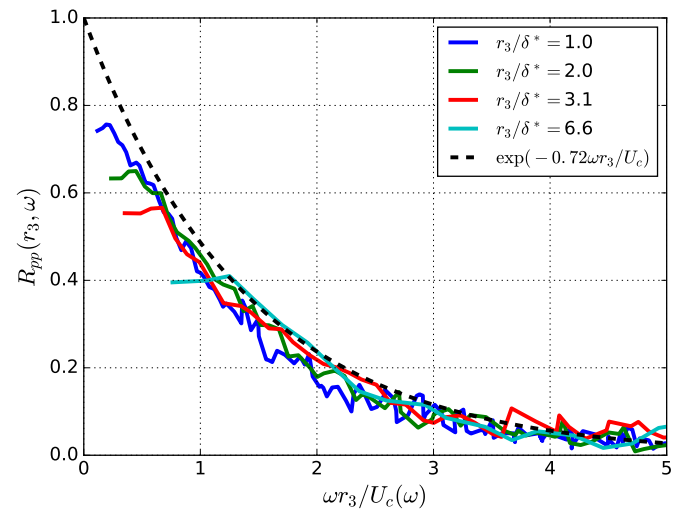

(e)

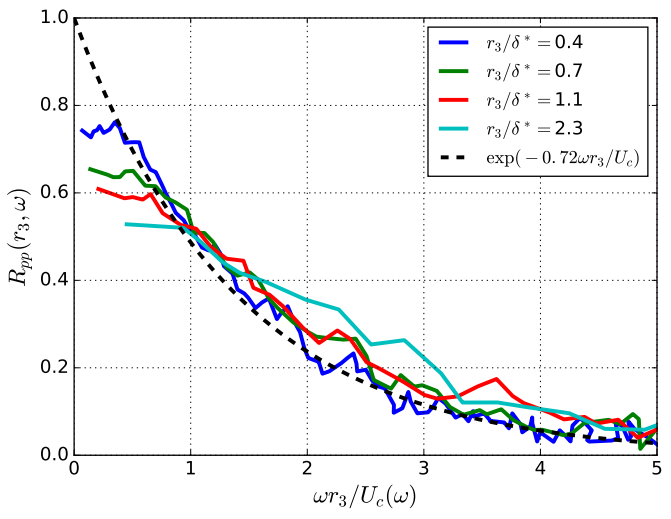

(b)

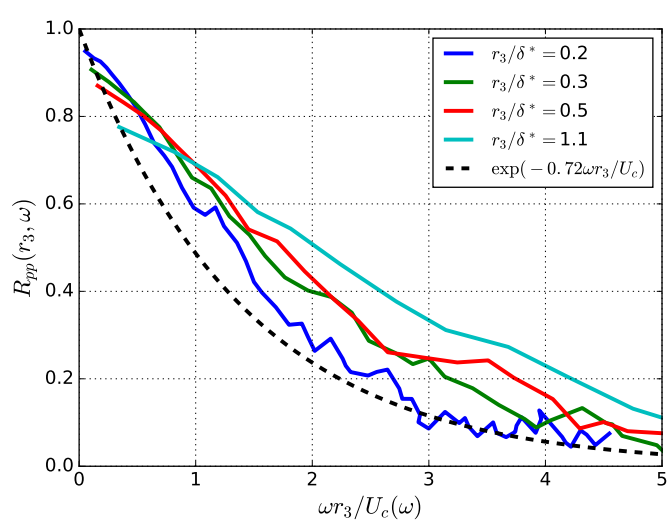

(d)

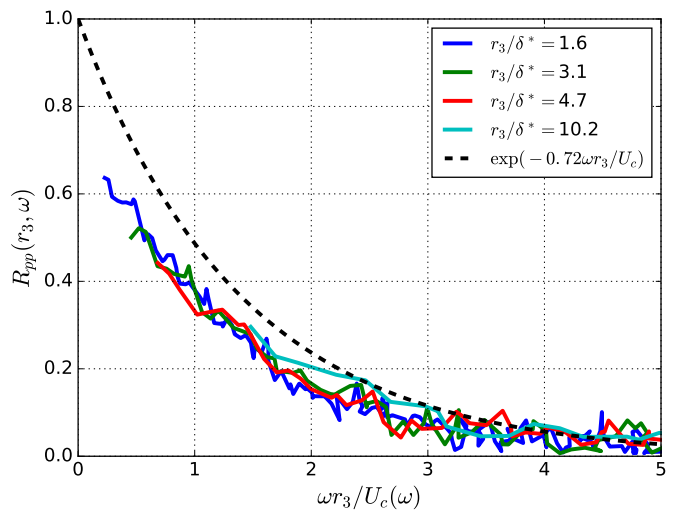

(f)

Figure 15: Lateral coherence as a function of $\omega r_{3} / U_{c}(\omega)$ at the freestream velocity of $30.2 \mathrm{~m} / \mathrm{s}$; (a) ZPG; (b) APG $-6^{\circ}$; (c) APG $-10^{\circ}$; (d) APG $-14^{\circ}$; (e) FPG $12^{\circ}$; (f) FPG $14^{\circ}$.

convecting downstream in the boundary layer. Similar observations were reported by Schloemer, ${ }^{8}$ Brooks \& Hodgson ${ }^{31}$ and Catlett et al. ${ }^{11}$ Note that even for the strongest initial APG (APG $-14^{\circ}$ case) the longitudinal coherence shows the similarity scaling behavior, although the trend can be not necessarily characterized using a single exponential function. The larger frequencies share a stronger decay rate (a larger constant for the exponential function), this feature may be related to the different phase velocity trends from low frequencies to higher frequencies between ZPG and APG boundary layers, see Fig. 16. The phase velocity of the APG boundary layers shows that the velocity at higher frequencies is much smaller than at lower frequencies compared to the ZPG boundary layer, and a smaller velocity means a longer travel time over a constant distance which could cause a larger decay. Figs. 14(e-f) show a slightly 
increased longitudinal coherence in FPG boundary layers.

Figs. 15(b-f) show a smaller decay rate for the lateral coherence in an APG boundary layer and a larger decay rate in a FPG boundary layer. Note that the coherence curves collapse in FPG boundary layers but not any more in strong APG boundary layers, e.g. the $\mathrm{APG}-10^{\circ}$ and $\mathrm{APG}-14^{\circ}$ cases.

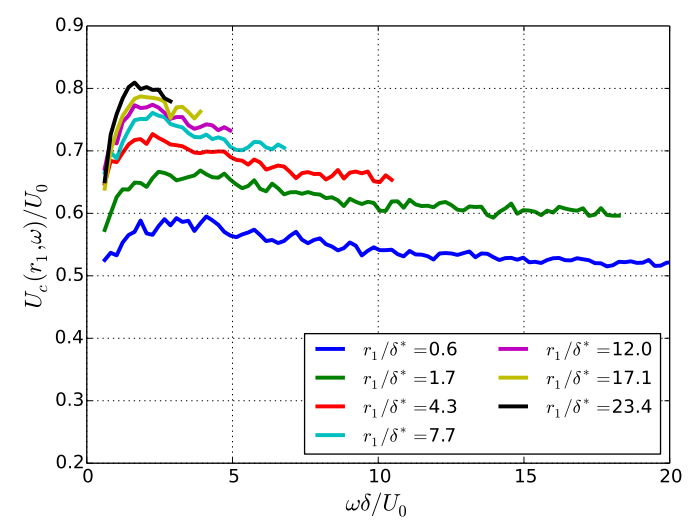

(a)

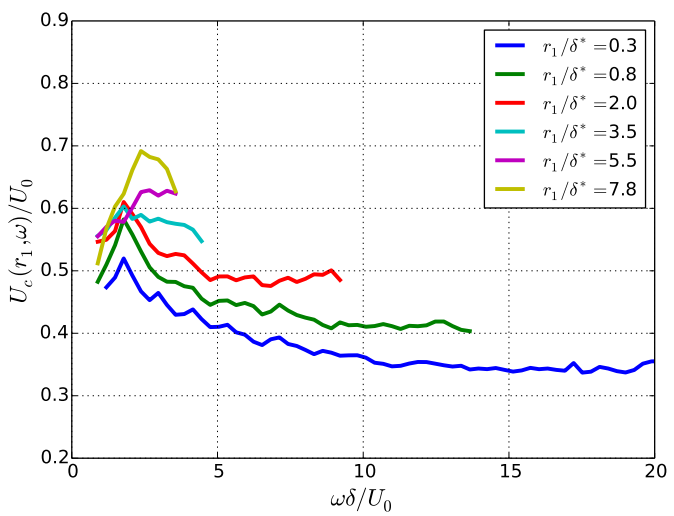

(c)

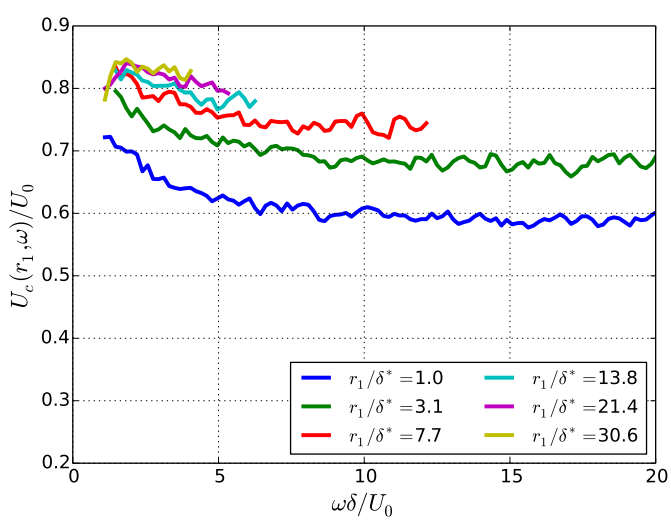

(e)

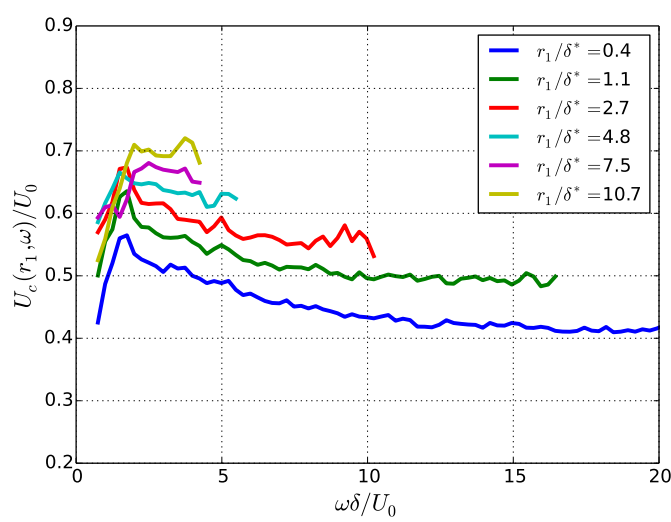

(b)

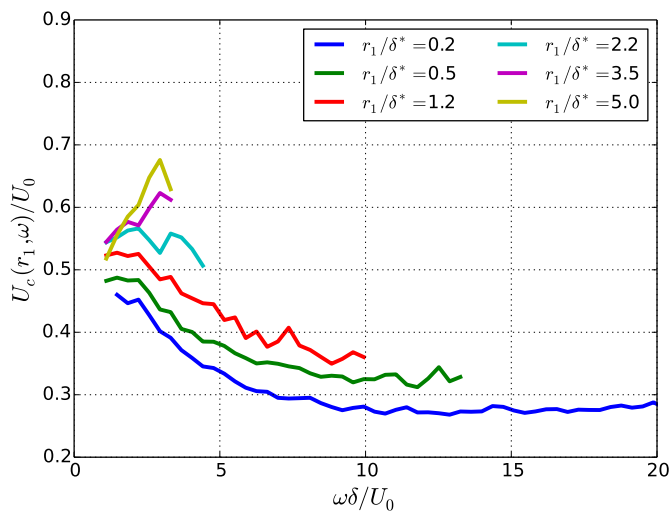

(d)

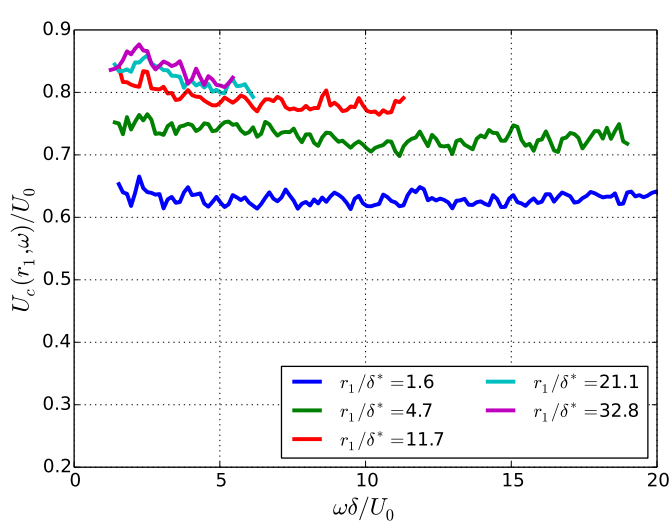

(f)

Figure 16: Phase velocities as a function of $\omega \delta / U_{c}\left(r_{1}, \omega\right)$; (a) ZPG; (b) APG $-6^{\circ}$; (c) APG $-10^{\circ}$; (d) APG $-14^{\circ}$; (e) FPG $12^{\circ}$; (f) FPG $14^{\circ}$.

Fig. 16 shows the convective phase velocities at the freestream velocity of $30.2 \mathrm{~m} / \mathrm{s}$ for ZPG, APG and FPG boundary layers. A larger velocity is measured at a larger longitudinal distance for all the configurations. This is because the smaller eddies close to the wall moving with a slower velocity decay faster as convecting downstream, thus at the larger distance the velocity is rather attributed to the larger eddies moving with a higher velocity. It is found that the obtained velocity decreases for an APG boundary layer compared to the ZPG boundary layer while it increases for an FPG boundary layer. This 
trend is primarily as a result of the different mean flow velocity profile shape for the boundary layers. The important portion $y<0.5 \delta$ in the boundary layer for the wall pressure fluctuations owns a larger mean flow velocity $u / U_{0}$ for the FPG boundary layer than the APG boundary layer, see Fig. 6(a). The smallest mean flow velocity in this portion is found in the APG $-14^{\circ}$ case with the smallest convective velocities.

Another view to the convective features can be drawn from the mean convective velocity $\bar{U}_{c}$, which can be obtained by using the time shift $\tau$ of the maximum correlation for a fixed longitudinal separation $r_{1}, \bar{U}_{c}\left(r_{1}\right)=r_{1} / \tau\left(r_{1}\right)$. The results for ZPG, APG and FPG boundary layers at the freestream velocity of $30.2 \mathrm{~m} / \mathrm{s}$ are shown in Fig. 17. It shows the same trend as obtained from the phase velocity which a FPG boundary layer owns a faster convective velocity while an APG boundary layer a slower. The maximum mean convective velocity approaches $0.82 U_{0}$ for the FPG boundary layer and $<0.7 U_{0}$ for the strong APG boundary layer of $14^{\circ}$. The maximum mean convective velocity implies the position of the 'longest lived' eddies in the boundary layer, if we assume obtained velocity origins from the region of the boundary layer where owns the same velocity. It is found that the position of the 'longest lived' eddies moves far away from the wall from a FPG boundary layer to an APG boundary, see Fig. 6(a).

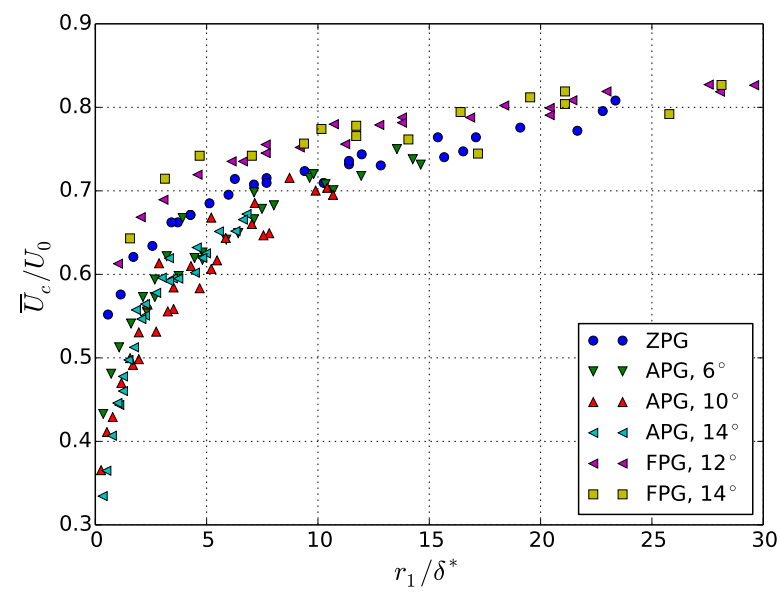

Figure 17: Mean convective velocity $\bar{U}_{c}$ at the freestream velocity of $30.2 \mathrm{~m} / \mathrm{s}$.

\section{Conclusion}

The wall pressure fluctuations were measured on a flat plate beneath zero pressure gradient (ZPG), adverse pressure gradient (APG), and favorable pressure gradient (FPG) boundary layers. The APG and FPG boundary layers were realized with a rotatable NACA 0012 airfoil. Mean flow properties of the boundary layers were measured by using hot wires. Twelve subminiature pressure sensors were placed in longitudinal- and lateral directions to measure both the one-point spectra and the two-point statistics of the fluctuating pressure field.

The level of the one-point spectra increases for an APG boundary layer and the maximum location of the spectra shifts to a lower frequency when compared to a ZPG. The slope of the mid-frequency roll-off becomes steeper for an APG boundary layer, whereas it tends to flatten for a FPG boundary layer. The APG spectra collapse by using the scaling variables $u_{\tau} / Q^{2} \theta$ and $\omega \theta / U_{0}$. It is found that the slope of the mid-frequency decrease in level can be well characterized by the boundary layer shape factor $H$. An empirical spectral model for the APG spectra is proposed based on the measured data. The predictions from the present model are compared to the present measured data and other two experimental results from literature. A good agreement is obtained.

The effects of the pressure gradient on the cross-spectra and the convective velocities are discussed. For APG boundary layer the coherence decay rate is increased in longitudinal direction and decreased in lateral direction, when compared to ZPG boundary layers. The effect is the larger the stronger APG. Only a slight decrease of the decay rate in longitudinal direction and increase in lateral direction is found for a FPG boundary layer. The convective velocities become slower for an APG boundary layer and faster for a FPG boundary layer. 


\section{Acknowledgments}

This work was conducted in the framework of the DLR project CENT.

\section{References}

${ }^{1}$ Blake, W. K., Mechanics of flow-induced sound and vibration, Academic Press, Inc., 1986.

${ }^{2}$ Willmarth, W. W. and Wooldridge, C. E., "Measurements of the fluctuating pressure at the wall beneath a thick turbulent boundary layer." J. Fluid Mech., Vol. 14, 1962, pp. 187-210.

${ }^{3}$ Bull, M. K., "Wall pressure fluctuations associated with subsonic turbulent boundary layer flow." J. Fluid Mech., Vol. 28, 1967, pp. 719-754.

${ }^{4}$ Blake, W. K., "Turbulent boundary layer wall pressure fluctuations on smooth or rough walls." J. Fluid Mech., Vol. 44(4), 1970, pp. 637-660.

${ }^{5}$ Farabee, T. M. and Casarella, M. J., "Spectral features of wall pressure fluctuations beneath turbulent boundary layers." Phys. Fluids, Vol. A3(10), 1991, pp. 2410-2420.

${ }^{6}$ Goody, M., "Empirical spectral model of surface pressure fluctuations." AIAA Journal, Vol. 42, No.9, 2004.

${ }^{7}$ Corcos, G. M., "The structure of the turbulent pressure field in boundary layer flows." J. Fluid Mech., Vol. 18, 1964, pp. $353-378$.

${ }^{8}$ Schloemer, H. H., "Effects of pressure gradients on turbulent-boundary-Layer wall-pressure fluctuations." J. Acoust. Soc. Am., Vol. 42(1), 1967, pp. 93-113.

${ }^{9}$ Rozenberg, Y., Robert, G., and Moreau, S., "Wall-pressure spectral model including the adverse pressure gradient effects." AIAA Journal, Vol. 50(10), 2012, pp. 2168-2179.

${ }^{10}$ Catlett, M. R., Forest, J. B., Anderson, J. M., and Stewart, D. O., "Empirical spectral model of surface pressure fluctuations beneath adverse pressure gradients." AIAA Paper, 2014.

${ }^{11}$ Catlett, M. R., Anderson, J. M., Forest, J. B., and Stewart, D. O., "Empirical modeling of pressure spectra in adverse pressure gradient turbulent boundary layers." AIAA Journal, Vol. 54(2), 2016.

${ }^{12}$ Suryadi, A. and Herr, M., "Wall pressure spectra on a DU96-W-180 profile from low to pre-stall angles of attack." AIAA Paper, 2015.

${ }^{13}$ Clauser, F. H., "Turbulent boundary layers in adverse pressure gradients." Journal of the Aeronautical Sciences, Vol. 21(2), 1954, pp. 91-108. 253.

${ }^{14}$ Mellor, G. L. and Gibson, D. M., "Equilibrium turbulent boundary layers." J. Fluid Mech., Vol. 24(2), 1966, pp. 225-

${ }^{15}$ Herring, H. J. and Norbury, J. F., "Some experiments on equilibrium turbulent boundary layers in favorable pressure gradients." J. Fluid Mech., Vol. 27(3), 1967, pp. 541-549.

${ }^{16}$ Narasimha, R. and Prasad, S. N., "Leading edge shape for flat plate boundary layer studies." Experiments in Fluids, Vol. 17(5), 1994, pp. 358-360.

${ }^{17}$ Mosallem, M. M., "Numerical and experimental investigation of beveled trailing edge flow fields." Journal of Hydrodynamics, Vol. 20(3), 2008, pp. 273-279.

${ }^{18}$ Spalding, D. B., "A single formula for the law of the wall." J. Appl. Mech., Vol. 28, 1961, pp. 455-457.

${ }^{19}$ Coles, D. E. and Hirst, E. A., "Computation of turbulent boundary layers." AFOSRIFP Stanford Conference, Vol. 2, 1968.

${ }^{20}$ White, F. M., Viscous fluid flow, McGraw-Hill Inc., 1991

${ }^{21}$ Nikuradse, J., "Turbulente Reibungsschichten an der Platte." ZWB, R.Oldenbourg, München and Berlin, 1942.

${ }^{22}$ Leclerco, D. J. J. and Bohineust, X., "Modeling the wave-vector frequency spectrum of turbulent boundary layer wall pressure." J. Sound Vib., Vol. 257(3), 2002, pp. 477-501.

${ }^{23}$ Panton, R. L. and Linebarger, J. H., "Wall pressure spectra for equilibrium boundary layers." J. Fluid Mech., Vol. 65, 1974, pp. 261-287.

${ }^{24} \mathrm{Hu}, \mathrm{N}$. , Reiche, N., and Ewert, R., "Simulation of turbulent boundary layer wall pressure fluctuations via Poisson equation and synthetic turbulence." (submitted to J. Fluid Mech., 2016).

${ }^{25} \mathrm{Hu}, \mathrm{N} .$, Appel, C., Herr, M., Reiche, N., and Ewert, R., "Numerical study of Wall pressure fluctuations for zero and non-zero pressure gradient turbulent boundary layers." AIAA Paper, 2016.

${ }^{26}$ Howe, M. S., Acoustics of fluid-structure interactions, Cambridge Univ. Press, 1998.

${ }^{27}$ Kraichnan, R. H., "Pressure fluctuations in turbulent flow over a flat plate." J. Acoust. Soc. Am., Vol. 28(3), 1956, pp. 378-390.

${ }^{28}$ Meecham, W. C. and Tavis, M. T., "Theoretical pressure correlation functions in turbulent boundary layer." Phys. Fluids, Vol. 23, 1980, pp. 1119-1131.

${ }^{29}$ Hodgson, T. H., Pressure fluctuations in shear flow turbulence, Ph.D. thesis, University of London, 1962.

${ }^{30}$ Corcos, G. M., "Resolution of pressure in turbulence." J. Acoust. Soc. Am., Vol. 35, 1964, pp. 192-199.

${ }^{31}$ Brooks, T. F. and Hodgson, T. H., "Trailing edge noise prediction from measured surface pressure." J. Sound Vib., Vol. 78(1), 1981, pp. 69-117. 